\title{
Influence of Magnetic Field and Thermal Radiation on Steady Free Convective Flow in a Porous Medium
}

\author{
A. S. Idowu, B. O. Falodun \\ Department of Mathematics, University of Ilorin, Ilorin, Kwara State, Nigeria.
}

\begin{abstract}
This paper investigated the influence of magnetic field and thermal radiation on steady free convective flow embedded in a porous medium with Soret effects. The governing equations are systems of nonlinear partial differential equations. Similarity variables were introduced on the set of governing equations and it was transformed into set of coupled nonlinear ordinary differential equations. The transformed coupled nonlinear ordinary differential equations are solved using the Spectral Homotopy Analysis Method (SHAM). Influence of pertinent flow parameters is displayed in figures and table. Comparisons were done with existing problems/models in literature and our results were in good agreement. The results revealed that as the magnetic parameter increased the velocity profile was found to decrease as a result of the magnetic field applied in the direction of the flow but negligible or has no effect on the velocity distribution. The results shows that increase in the suction/injection velocity and Magnetic field parameter enrich the temperature and concentration.
\end{abstract}

KEYWORDS: Buoyancy; Free Convective Flow; Magnetic Field; Porous Medium; Soret; Suction/Injection.

[Received September 10 2017; Revised February 28 2018; Accepted March 24 2018] Print ISSN: 0189-9546 | Online ISSN: 2437-2110

\begin{tabular}{ll}
\multicolumn{3}{c}{ NOMENCLATURE } \\
$a, b$ stratification rate of the gradient of & Greek symbols \\
ambient temperature and concentration & $v$ kinematic viscosity \\
profiles respectively & $\rho$ fluid density \\
$B$ constant & $\gamma \quad$ heat generation/absorption \\
$C$ dimensional concentration & $\sigma \quad$ electrical conductivity \\
$g$ acceleration due to gravity & $\eta \quad$ similarity variable \\
$x$ dimensional coordinate along the vertical & $\beta_{t}$ thermal expansion coefficient \\
porous plate & $\beta_{c}$ concentration expansion coefficient \\
$y$ dimensional coordinate normal to the & $\alpha$ fluid thermal diffusivity \\
porous plate & $f(\eta)$ dimensionless stream function \\
$T$ dimensional temperature & $\theta(\eta)$ dimensionless temperature \\
$u$ velocity component in x direction & $\phi(\eta)$ dimensionless species concentration \\
$v$ velocity component in y direction &
\end{tabular}

\section{Superscripts}

$R^{*}$ chemical reaction parameter

$k^{\prime} \quad$ permeability term

\section{Subscripts}

$T_{m}$ mean fluid temperature

$c_{p} \quad$ specific heat at constant pressure

$B_{0} \quad$ externally imposed magnetic field

$D_{m} \quad$ Mass diffusivity

$k_{T}$ thermal diffusivity ratio

$c_{s}$ concentration susceptivity

$q_{r}$ radiative heat flux

$T_{\infty}$ free stream temperature 


\section{INTRODUCTION}

The phenomenon of free convective flow in porous medium is of great importance in our day to day activities because of its numerous applications in engineering. This phenomenon is applicable in food processing and crystal growth. Similarity transformations of heat and mass transfer effects on steady MHD free convection dissipative fluid flow past an inclined porous surface with chemical reaction was investigated by Reddy et al. (2014). Their coupled ordinary differential equations are solved numerically using RungeKutta method along with shooting technique. Their result shows that increase in the Schmidt number gradually reduces the velocity and concentration profiles.

Also, they found out that the velocity and concentration decreases as the chemical reaction is increased. Ibrahim (2014) studied unsteady MHD free convective flow along a vertical porous plate embedded in a porous medium with heat generation, variable suction and chemical reaction effects. In their analysis, similarity transformations are used to reduce the governing partial differential equations to a system of self-similar equations and solved using Runge-Kutta method along with shooting technique. Their finding shows that as the Schmidt number or chemical reaction parameter increases both the velocity and concentration profiles decreases.

Mohamed et al. (2010) investigated thermal radiation and MHD effects on free convective flow of a polar fluid through a porous medium in the presence of internal heat generation and chemical reaction. Their coupled nonlinear differential equations are solved using a two-term perturbation method. They used Eckert number as a perturbation parameter and found out that the Nusselt number increases with an increase in Prandtl number and radiation parameter but decreases as Magnetic parameter increases with values of heat generation parameter. Rushikumai and Gangadhar (2012) studied MHD free convection flow between two parallel porous walls with varying temperature. In their analysis, similarity variable was used to reduce the partial differential equations into ordinary differential equations. Their reduced equations were solved using shooting method.

They found out that the temperature increases with an increase in the Magnetic field parameter. Also, their result shows that the velocity decreases as the Magnetic field parameter, permeability parameter and Prandtl number increases. Kalyani et al. (2015) discussed MHD mixed convection flow past a vertical porous plate in a porous medium with heat source/sink and Soret effects. In their analysis, they use similarity transformations to reduce the governing partial differential equations into non-linear ordinary differential equations. Their transformed equations were then solved using the implicit finite difference scheme known as Keller Box method. Their finding reveals that velocity and concentration profiles increase when Soret number is increased. The Soret effect on free convective unsteady MHD flow over a vertical plate with heat source was investigated by Bhavana et al. (2013). They solved their dimensionless governing equations analytically with the help of perturbation technique.

They found out that an increase in the radiation parameter increases the skin friction coefficients. Prakash et al. (2016) discussed diffusion-thermo effects on MHD free convective radiative and chemically reactive boundary layer flow through a porous medium over a vertical plate. In their study, they employed perturbation method in solving the nonlinear coupled partial differential equations. It was found out in their study that the thermal and momentum boundary layer thickness is increased with diffusion-thermo parameter. Subhakar and Gangadhar (2012) studied Soret and Dufour effects on MHD free convection heat and mass transfer flow over a stretching vertical plate with suction and heat source/sink.

When the thermal condition of a fluid is at high temperature, the effect of thermal radiation is of great significance. Many engineering problems occur at higher temperature. Despite the role of thermal radiation on fluid flow, it is unfortunate that many researcher's in the field of fluid dynamics neglect its effect in their investigations. Alao et al. (2016) investigated thermal radiation, Soret and Dufour effects on an unsteady heat and mass transfer flow of a chemically reacting fluid past a semi-infinite vertical plate with viscous dissipation. In their study, they introduced nondimensional quantities on their governing partial differential equations and solved the transformed dimensionless equations numerically using the spectral relaxation method.

Their result shows that increasing the thermal radiation parameter decreases the temperature distribution with a cooled plate. Rajput and Kumar (2012) investigated radiation effects on MHD flow past an impulsively started vertical plate with variable heat and mass transfer. In their analysis, they solved their governing equations using the Laplacetransform technique. They found out that increase in the radiation parameter, Magnetic field parameter and Prandtl number decreases the velocity. Mahdy (2008) studied the effects of radiation on unsteady MHD convective heat transfer past a semi-infinite vertical porous moving surface with variable suction. In the study, perturbation technique is used to transform the governing equations into nonlinear ordinary differential equations and later solved using the shooting method. The result revealed that the temperature, skin friction and heat transfer increase as the radiation parameter is increased.

Olanrewaju (2012) reported similarity solution for natural convection from a moving vertical plate with internal heat generation and a convective boundary condition in the presence of thermal radiation and viscous dissipation. In the study, similarity variable is used to transform the governing non-linear partial differential equations into a system of ordinary differential equations. The transformed equations are solved using shooting iteration technique with sixth order Runge-Kutta integration scheme. It was found out in the study that the thermal radiation, Eckert number and internal heat generation oppose the flow of heat from the left surface 
to right except the Grashof number is strong to convert both the internally generated heat in the fluid.

Double diffusive flow plays an important role in engineering processes. The important areas of application are chemical catalytic reactors and processes, petroleum reservoirs, nuclear waste disposals, etc. The buoyancy due to temperature and concentration gradients is responsible for diffusive flow. Whenever heat and mass transfer occur at the same time in a moving fluid, the relations between the driving capacity and the energy fluxes which are generated by the action of both temperature and concentration gradients are more complicated. When the energy flux is caused by composition gradient, we have the diffusion-thermo or Dufour effect. The Dufour effect is found on the energy equation.

Mass fluxes are also created by temperature gradient and this is called thermo-diffusion or Soret effects. The effect of soret is also found in the species equation. The effects of Soret and Dufour are mostly neglected in literature because they are of smaller order of magnitude than the effects recommended by Fick's laws. The Soret effect has been utilized in isotope separation. Sharma and Aich (2016) studied Soret and Dufour effects on steady MHD flow in presence of heat source through a porous medium over a nonisothermal stretching sheet. Their transformed ordinary differential equations are solved numerically using bvp4c solver of MATLAB and they found out that the rate of heat transfer decreases in magnitude with increase in permeability parameter, magnetic field parameter, heat source parameter and Dufour number.

Dufour and Soret effects on steady MHD free convective flow past a vertical porous plate embedded in a porous medium with chemical reaction, radiation, heat generation and viscous dissipation has been presented. Their transformed equations are solved numerically by employing shooting method along with fourth-order Runge-Kutta integration scheme. Their result revealed that increase in the radiation parameter decreases the velocity profile. In another development, Kala et al. (2017) studied diffusion-thermo and thermo-diffusion effects on MHD fluid flow over nonlinearly stretching sheet through a Non-Darcy porous medium. In their study, they employed similarity transformations to reduce the governing equations into ordinary differential equations. The transformed equations are then solved with bvp4c MATLAB. They found out that the Sherwood number increases as the Dufour number increases.

Effect of magnetic field on free convective flow is of great importance in plasma studies amd MHD power generator. The importance of magnetic field has been discussed extensively in literature. Influence of magnetic field, viscous dissipation and thermophoresis on DarcyForcheimer mixed convection flow in fluid saturated porous media was investigated by Fagbade et al. (2015). In their analysis, they used similarity variable to reduce the governing partial differential equations into coupled ordinary differential equations and solved with the help of spectral homotopy analysis method. They reported that Magnetic field can control the characteristics flow of heat and mass transfer.
Sravanthi et al. (2013) presented thermo-diffusion and chemical reaction effects on a steady mixed convective heat and mass transfer flow with induced magnetic field. Their dimensionless governing equations are solved using series solution technique and it was found out that increasing the Soret number result to a reduction in the values of induced Magnetic field. In 2010 Ibrahim and Makinde presented chemically reacting MHD boundary layer flow of heat and mass transfer over a moving vertical plate with suction. Similarity transformations are used on their governing partial differential equations to have non-linear ordinary differential equations.

Their transformed non-linear ordinary differential equations are solved using shooting techniques with the fourth order Runge-Kutta integration scheme. Their result revealed that the momentum boundary layer thickness decreases and both thermal and concentration boundary layer thickness increase with an increase in the Magnetic field intensity. Also, in the year 2011 they considered chemically reacting magnetohydrodynamics (MHD) boundary layer flow of heat and mass transfer past a low-heat-resistance sheet moving vertically downwards. They solved their non-linear coupled differential equations using Runge-Kutta integration scheme coupled with Newton-Raphson shooting technique.

Their result shows that increase in the buoyancy force parameter increases the velocity but decreases the temperature due to convective cooling. Rao et al. (2014) extended the model of Ibrahim and Makinde (2010) by considering the Soret and Dufour effects on MHD boundary layer flow over a moving vertical porous plate with suction. In the analysis of Rao et al. (2014), Runge-Kutta fourth order shooting method was used to solve their equations of motion. Their findings revealed that as Dufour and Soret number increases the velocity increases. The effects of these two thermo-physical parameters were noted to have been neglected in the analysis of Ibrahim and Makinde (2010).

From all the literatures discussed above and also to the best of our knowledge, no study in the literature has discussed the influence of magnetic field and thermal radiation on steady free convective flow embedded in a porous medium with Soret and Dufour effects. Hence, the aim in this paper is to investigate the influence of magnetic field and thermal radiation on steady free convective flow embedded in a porous medium with soret and dufour effects. This paper employs the spectral method called spectral homotopy analysis method in solving the resulting equations of the problem under investigation. Spectral methods are now a common method used by many researchers in solving problems in sciences and engineering because of its high accuracy and easy computation. The spectral method employs the use of Chebyshev spectral collocation method in solving both partial and ordinary differential equations.

\section{FORMULATION OF THE PROBLEM}

In this paper, a steady two-dimensional laminar boundary layer flow of a viscous, incompressible, radiating fluid along a vertical plate is considered. The vertical plate and the medium are considered to be porous and the effect of viscous dissipation is taking into account while Joule heating effect is 
neglected (see Figure 1). The $\mathrm{x}$-axis is taken along the vertical porous plate in the upward direction while the $y$-axis is considered normal to the plate. The magnetic field is considered opposite to the flow and thereby considered negative. The level of concentration of foreign mass is assumed to be high and as a result of this, we cannot neglect the effects of thermo-diffusion and diffusion-thermo. However, using the Boussinesq's approximation, the fluid flow is governed by the equations below:

$\frac{\partial u}{\partial x}+\frac{\partial v}{\partial y}=0$

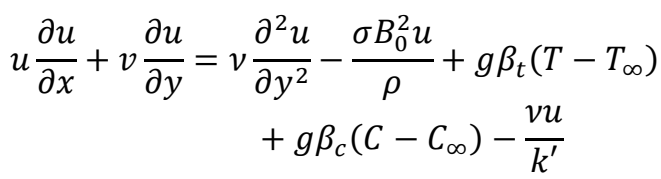

$u \frac{\partial T}{\partial x}+v \frac{\partial T}{\partial y}$

$=\alpha \frac{\partial^{2} T}{\partial y^{2}}-\frac{1}{\rho c_{p}} \frac{\partial q_{r}}{\partial y}+\frac{v}{c_{p}}\left(\frac{\partial u}{\partial y}\right)^{2}+\frac{D_{m} K_{T}}{c_{s} c_{p}} \frac{\partial^{2} C}{\partial y^{2}}$

$+\frac{Q_{0}\left(T-T_{\infty}\right)}{\rho c_{p}}$

$u \frac{\partial C}{\partial x}+v \frac{\partial C}{\partial y}=D_{m} \frac{\partial^{2} C}{\partial y^{2}}+\frac{D_{m} K_{T}}{T_{m}} \frac{\partial^{2} T}{\partial y^{2}}-R^{*}\left(C-C_{\infty}\right)$

subject to:

$$
\begin{aligned}
& v=V, u=B x, T=T_{w}=T_{\infty}+a x, \\
& C=C_{w}=C_{\infty}+b x \quad \text { at } y=0 \\
& u \rightarrow 0, T \rightarrow T_{\infty}, C \rightarrow C_{\infty} \text { a sy } \infty
\end{aligned}
$$

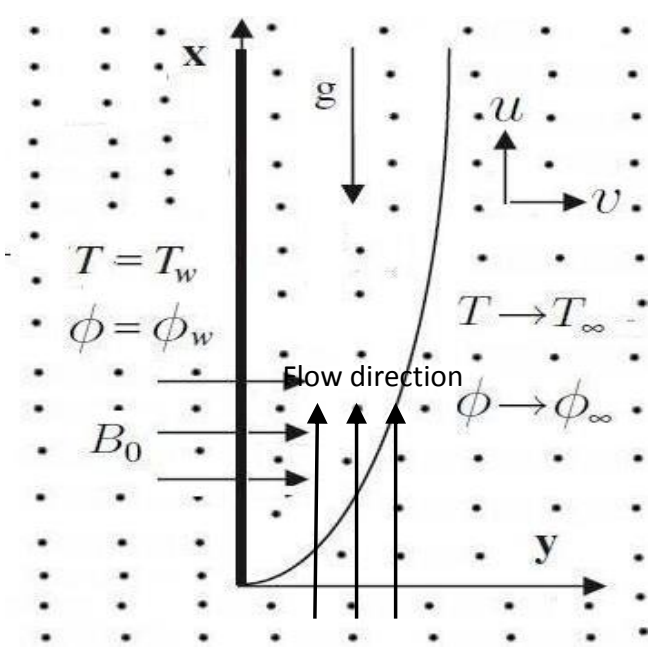

Figure 1: Physical geometry of the problem.
By defining the stream function $u=\frac{\partial \psi}{\partial y}$ and $v=-\frac{\partial \psi}{\partial x}$, the continuity equation become

$\frac{\partial^{2} \psi}{\partial x \partial y}-\frac{\partial^{2} \psi}{\partial y \partial x}=0$

Obviously the stream function satisfies the continuity equation (1).

The radiative heat flux is simplified using the Rosseland diffusion approximation and following Alao et al. (2016) we have the term $q_{r}$ in the energy equation given by

$q_{r}=-\frac{4 \sigma_{s}}{3 k_{e}} \frac{\partial T^{4}}{\partial y}$

where $\sigma_{s}$ is the Stefan-Boltzman constant and $k_{e}$ is the absorption coefficient. The present study is limited to an optically thin fluid by using the Rosseland approximation. If temperature difference within the flow is sufficiently small, Eq. (8) is linearized by expanding $T^{4}$ in Taylor series about $T_{\infty}$ and neglecting the higher order term gives:

$T^{4} \cong 4 T_{\infty}^{3} T-3 T_{\infty}^{4}$

Putting Eq. (8) and Eq. (9) into Eq. (3), we obtain

$$
\begin{aligned}
u \frac{\partial T}{\partial x}+v \frac{\partial T}{\partial y}=\alpha & \frac{\partial^{2} T}{\partial y^{2}}+\frac{16 \sigma_{s} T_{\infty}^{3}}{3 \rho c_{p} k_{e}} \frac{\partial^{2} T}{\partial y^{2}}+\frac{v}{c_{p}}\left(\frac{\partial u}{\partial y}\right)^{2} \\
& +\frac{D_{m} K_{T}}{c_{s} c_{p}} \frac{\partial^{2} C}{\partial y^{2}}+\frac{Q_{0}\left(T-T_{\infty}\right)}{\rho c_{p}}
\end{aligned}
$$

The following similarity transformations are introduced on the governing partial differential equations

$$
\begin{gathered}
\eta=\sqrt{\frac{B}{v}} y, \psi=x f(\eta) \sqrt{B v}, \theta(\eta)=\frac{T-T_{\infty}}{T_{w}-T_{\infty}}, \\
\phi(\eta)=\frac{C-C_{\infty}}{C_{w}-C_{\infty}}
\end{gathered}
$$

Utilizing the above similarity transformations on the governing partial differential equations leads to:

$$
\begin{aligned}
& f^{\prime \prime \prime}+f f^{\prime \prime}-f^{\prime} f^{\prime}-\left(M+\frac{1}{k_{0}}\right) f^{\prime}+\operatorname{Gr} \theta+G c \phi=0 \\
& \begin{array}{c}
\left(1+\frac{4}{3} N r\right) \theta^{\prime \prime}+\operatorname{Pr} f \theta^{\prime}-\operatorname{Pr} f^{\prime} \theta+\operatorname{Pr} D u \phi^{\prime \prime}+\operatorname{Pr} \gamma \theta \\
\quad+\operatorname{PrEc} f^{\prime} f^{\prime}=0
\end{array} \\
& \begin{array}{c}
\phi^{\prime \prime}+S c f \phi^{\prime}-S c f^{\prime} \phi+S c S r \theta^{\prime \prime}-S c k_{r}^{2} \phi \\
\text { Subject to the following boundary conditions }
\end{array} \\
& f(\eta)=-f_{w}, f^{\prime}(\eta)=1, \theta(\eta)=1, \phi(\eta)=1 \text { at } \eta=0 \\
& f^{\prime}(\eta)=0, \theta(\eta)=0, \phi(\eta)=0 \text { as } \eta \rightarrow \infty
\end{aligned}
$$

Where $M, k_{0}, G r, G c, N r, P r, D u, \gamma, E c, S c, S r, k_{r}^{2}, f w$ are the Magnetic field parameter, permeability term, thermal Grashof number, mass Grashof number, radiation parameter, Prandtl number, Dufour number, heat generation parameter, Eckert number, Schmidtl number, Soret number, chemical reaction parameter, suction/injection velocity and they are defined as: 


$$
\begin{gathered}
M=\frac{\sigma B_{0}^{2}}{\rho B}, \quad G r=\frac{g \beta_{t}\left(T_{w}-T_{\infty}\right)}{x B^{2}}, \\
G c=\frac{g \beta_{c}\left(C_{w}-C_{\infty}\right)}{x B^{2}}, \quad k_{0}=\frac{k^{\prime} B}{v}, \\
N r=\frac{4 \sigma_{s} T_{\infty}^{3}}{k_{e} k}, S c=\frac{v}{D_{m}} \\
D u=\frac{D_{m} k_{T}\left(C_{w}-C_{\infty}\right)}{c_{s} c_{p}\left(T_{w}-T_{\infty}\right)}, \\
S r=\frac{D_{m} k_{T}\left(T_{w}-T_{\infty}\right)}{T_{m} v\left(T_{w}-T_{\infty}\right)}, k_{r}=\frac{R^{*} v}{B^{2}}, P r \\
=\frac{v \rho c_{p}}{k}, E c=\frac{x^{2}}{c_{p}\left(T_{w}-T_{\infty}\right)}, f w \\
=-\frac{v_{w}(x)}{\sqrt{B v}}
\end{gathered}
$$

The physical quantities of engineering interest in this paper they are the skin friction coefficient (Cf), Nusselt number $\mathrm{Nu}$ ) and the Sherwood number (Sh). The physical quantities can be computed without any difficulty. The dimensionless form of these quantities is given as:

$C f=f^{\prime \prime}(0), N u=-\theta^{\prime}(0), S h=-\phi^{\prime}(0)$

\section{SPECTRAL HOMOTOPY ANALYSIS METHOD (SHAM)}

This paper employs the numerical version of the homotopy analysis method called Spectral homotopy analysis method in solving the set of coupled nonlinear ordinary differential equations that models the problem of free convective flow embedded in a porous medium. Detailed description and applications of the homotopy analysis method can be found in Liao (2003) and Liao (2012) solely written by Liao S.J. and he is credited for developing this method. However, the numerical version of HAM called SHAM is discussed extensively in Motsa et al. (2010). The SHAM combines HAM with the Chebyshev spectral collocation method in solving systems of ordinary differential equations. Spectral methods are now preferred by researchers' in solving ordinary differential equations and partial differential equations because of their high accuracy and ease computation.

Furthermore, in the spectral homotopy analysis method, the domain of the problem is transformed from $[0, L]$ to $[-1,1]$ using the algebraic mapping given by

$\xi=\frac{2 \eta}{L}-1 \quad \xi$

$\in[-1,1]$

For convenience we make the boundary conditions homogeneous by invoking the following transformation into Eq. (12)-Eqn .(16)

$f(\eta)=f(\xi)+f_{0}(\eta) ; \theta(\eta)=\theta(\xi)+\theta_{0}(\eta) ; \quad \phi(\eta)=$ $\phi(\xi)+\phi_{0}(\eta)$

Using Eq.(17)-Eq.(18) on the transformed governing equations and boundary conditions (12)-(16) yields:

$$
\begin{gathered}
f^{\prime \prime \prime}+f f^{\prime \prime}+d_{1} f+d_{2} f^{\prime \prime}-f^{\prime} f^{\prime}+d_{3} f^{\prime}-\left(M+\frac{1}{k_{0}}\right) f^{\prime} \\
+G r \theta+G c \phi=H_{1}(\eta)
\end{gathered}
$$

$$
\begin{aligned}
& \left(1+\frac{4}{3} N r\right) \theta^{\prime \prime}+\operatorname{Pr} f \theta^{\prime}+e_{1} f+e_{2} \theta^{\prime}-\operatorname{Pr} f^{\prime} \theta+e_{3} f^{\prime} \\
& +e_{4} \theta+\operatorname{Pr} D u \phi^{\prime}+\operatorname{Pr} \gamma \theta+\operatorname{Pr} E c f^{\prime} f^{\prime}+e_{5} f^{\prime} \\
& =H_{2}(\eta) \\
& \phi^{\prime \prime}+S c f \phi^{\prime}+g_{1} f+g_{2} \phi^{\prime}-S c f^{\prime} \phi-S c f^{\prime} \\
& +g_{3} \phi+S c S r \theta^{\prime \prime}+g_{4} \phi-S c k_{r}^{2} \phi=H_{3}(\eta)
\end{aligned}
$$$$
\text { Subject to: }
$$$$
f(-1)=f(1)=f^{\prime}(-1)=f^{\prime}(1)=0 ; \theta(-1)=\theta(1)=
$$$$
0 ; \phi(-1)=\phi(1)=0
$$

The coefficients of parameters

$d_{1}, d_{2}, d_{3}, e_{1}, e_{2}, e_{3}, e_{4}, e_{5}, g_{1}, g_{2}$ and $g_{3}$ are defined as:

$d_{1}=f_{0}^{\prime \prime}(\eta), d_{2}=f_{0}(\eta), d_{3}=-2 f_{0}^{\prime}(\eta)$,

$e_{1}=\operatorname{Pr} \theta_{0}^{\prime}(\eta), e_{2}=\operatorname{Pr}_{0}(\eta), e_{3}=\operatorname{Pr} \theta_{0}(\eta)$,

$e_{4}=-\operatorname{Pr} f_{0}^{\prime}(\eta), e_{5}=2 \operatorname{PrEc} f_{0}^{\prime}(\eta)$,

$g_{1}=S c \phi_{0}^{\prime}(\eta), g_{2}=S c f_{0}(\eta), g_{3}=-S c \phi_{0}(\eta)$,

$g_{4}=-S c f_{0}^{\prime}(\eta)$

And the term $H_{1}(\eta), H_{2}(\eta)$ and $H_{3}(\eta)$ are given by:

$H_{1}(\eta)=-f_{0}^{\prime \prime \prime}(\eta)-f_{0}(\eta) f_{0}^{\prime \prime}(\eta)+f_{0}^{\prime}(\eta) f_{0}^{\prime}(\eta)$

$$
\begin{aligned}
& +\left(M+\frac{1}{k_{0}}\right) f_{0}^{\prime}(\eta)-\operatorname{Gr} \theta_{0}(\eta) \\
& -\operatorname{Gc} \phi_{0}(\eta) \\
& H_{2}(\eta)=-\left(1+\frac{4}{3} N r\right) \theta_{0}^{\prime \prime}(\eta)-\operatorname{Pr} f_{0}(\eta) \theta_{0}^{\prime}(\eta) \\
& +\operatorname{Pr}_{0}^{\prime}(\eta) \theta_{0}(\eta)-\operatorname{PrDu} \phi_{0}^{\prime \prime}(\eta) \\
& -\operatorname{Pr} \theta_{0}(\eta)-\operatorname{PrEc} f_{0}^{\prime}(\eta) f_{0}^{\prime}(\eta) \\
& H_{3}(\eta)=-\phi_{0}^{\prime \prime}(\eta)-S c f_{0}(\eta) \phi_{0}^{\prime}(\eta)+S c f_{0}^{\prime}(\eta) \phi_{0}(\eta) \\
& -\operatorname{ScSr} \theta_{0}^{\prime \prime}(\eta)+\operatorname{Sck} k_{r}^{2} \phi_{0}(\eta)
\end{aligned}
$$

The nonhomogeneous linear part of (19)-(21) is of the form

$$
f_{l}^{\prime \prime \prime}+d_{1} f_{l}+d_{2} f_{l}^{\prime \prime}+d_{3} f_{l}^{\prime}-\left(M+\frac{1}{k_{0}}\right) f_{l}^{\prime}+G r \theta_{l}+G c \phi_{l}
$$$$
=H_{1}(\eta)
$$

$$
\begin{gathered}
\left(1+\frac{4}{3} N r\right) \theta_{l}^{\prime \prime}+e_{1} f_{l}+e_{2} \theta_{l}^{\prime}+e_{3} f_{l}^{\prime}+e_{4} \theta_{l}+\operatorname{PrDu} \phi_{l}^{\prime} \\
+\operatorname{Pr} \gamma \theta_{l}+e_{5} f_{l}^{\prime}=H_{2}(\eta) \\
\phi_{l}^{\prime \prime}+g_{1} f_{l}+g_{2} \phi_{l}^{\prime}+g_{3} \phi_{l}+S c S r \theta_{l}^{\prime \prime}-S c k_{r}^{2} \phi_{l}+g_{4} \phi \\
=H_{3}(\eta)
\end{gathered}
$$

Subject to

$f_{l}(-1)=f_{l}^{\prime}(1)=0, \theta_{l}(-1)=\theta_{l}(1)=0, \phi_{l}(-1)=\phi_{l}(1)$ $=0$

is the solution of the Spectral Homotopy Analysis Method. We proceed to use Chebyshev pseudospectral method to solve (26)-(29). In the above equations $f_{l}(\xi), \theta_{l}(\xi)$ and $\phi_{l}(\xi)$ are unknown functions that are approximated as truncated series of chebyshev polynomials given by

$$
\begin{aligned}
& f_{l}(\xi) \cong f_{l}^{N}\left(\xi_{j}\right)+\sum_{m=0}^{N} f_{m}^{\wedge} T_{m}\left(\xi_{j}\right), j=0, \ldots, N \\
& \theta_{l}(\xi) \cong \theta_{l}^{N}\left(\xi_{j}\right)+\sum_{m=0}^{N} \theta_{m}^{\wedge} T_{m}\left(\xi_{j}\right), j=0, \ldots, N \\
& \phi_{l}(\xi) \cong \phi_{l}^{N}\left(\xi_{j}\right)+\sum_{m=0}^{N} \phi_{m}^{\wedge} T_{m}\left(\xi_{j}\right), j=0, \ldots, N
\end{aligned}
$$

Following Fagbade et al. (2015), the Gauss-Lobatto collocation points $\xi_{0}, \xi_{1}, \ldots, \xi_{N}$ and the derivatives of the unknown functions $f_{l}(\xi), \theta_{l}(\xi)$ and $\phi_{l}(\xi)$ are defined by

$\xi_{j}=\cos \left(\frac{\pi j}{N}\right), j=0,1, \ldots, N$ 


$$
\left.\begin{array}{l}
\frac{d^{r} f_{l}}{d \xi^{r}}=\sum_{m=0}^{N} D_{m j} f_{j}\left(\xi_{j}\right), \\
\frac{d^{r} \theta_{l}}{d \xi^{r}} \\
=\sum_{m=0}^{N} D_{m j} \theta_{j}\left(\xi_{j}\right), \\
\frac{d^{r} \phi_{l}}{d \xi^{r}}=\sum_{m=0}^{N} D_{m j} \phi_{j}\left(\xi_{j}\right),
\end{array}\right\}
$$

where $\boldsymbol{D}$ is the Chebyshev spectral differentiation matrix and $r$ is the order of differentiation. Putting (33)-(34) into (26)(29) produces

$B F_{L}=G$

Subject to:

$$
\begin{gathered}
f_{l}\left(\xi_{N}\right)=0, \sum_{m=0}^{N} D_{0, k} f_{l}\left(\xi_{k}\right)=0, \theta_{l}\left(\xi_{N}\right)=0, \theta_{l}\left(\xi_{0}\right) \\
=0, \phi_{l}\left(\xi_{N}\right)=0, \phi_{l}\left(\xi_{0}\right)=0
\end{gathered}
$$

And

$$
B=\left(\begin{array}{lll}
B_{11} & B_{12} & B_{13} \\
B_{21} & B_{22} & B_{23} \\
B_{31} & B_{32} & B_{33}
\end{array}\right)
$$

From Eqn.(35) and Eqn.(37) we have;

$$
\begin{aligned}
& B_{11}=D^{3}+d_{1}+d_{2} D^{2}+d_{3} D-\left(M+\frac{1}{k_{0}}\right) D, B_{11}=G r I \\
& B_{13}=G c I, B_{12}=e_{1}+e_{3} D+e_{5} D, \quad B_{23}=\operatorname{Pr} D u D, \\
& B_{31}=g_{1} I, B_{22}=\left(1+\frac{4}{3} N r\right) D^{2}+e_{2} D+e_{4} I+\operatorname{Pr} \gamma I \\
& B_{33}=D^{2}+g_{2} D+g_{3} I-S c k_{r}^{2} I+g_{4} I, B_{32}=S c S r D^{2} \\
& F_{L} \\
& =\left[f_{l}\left(\xi_{0}\right), \ldots, f_{l}\left(\xi_{N}\right), \theta_{l}\left(\xi_{0}\right), \ldots, \theta_{l}\left(\xi_{N}\right), \phi_{l}\left(\xi_{0}\right), \ldots, \phi_{l}\left(\xi_{N}\right)\right]^{T} \\
& G=\left[H_{1}\left(\eta_{0}\right), H_{1}\left(\eta_{1}\right), \ldots, H_{1}\left(\eta_{N}\right), H_{2}\left(\eta_{0}\right), H_{2}\left(\eta_{1}\right), \ldots, H_{2}\left(\eta_{N}\right),\right. \\
& \left.H_{3}\left(\eta_{0}\right), H_{3}\left(\eta_{1}\right), \ldots, H_{3}\left(\eta_{N}\right)\right] \\
& d_{i}=\operatorname{diag}\left(\left[d_{i}\left(\eta_{0}\right), d_{i}\left(\eta_{1}\right), \ldots, d_{i}\left(\eta_{N-1}\right), d_{i}\left(\eta_{N}\right)\right]\right) \\
& e_{i}=\operatorname{diag}\left(\left[e_{i}\left(\eta_{0}\right), e_{i}\left(\eta_{1}\right), \ldots, e_{i}\left(\eta_{N-1}\right), e_{i}\left(\eta_{N}\right)\right]\right) \\
& g_{i}=\operatorname{diag}\left(\left[g_{i}\left(\eta_{0}\right), g_{i}\left(\eta_{1}\right), \ldots, g_{i}\left(\eta_{N-1}\right), g_{i}\left(\eta_{N}\right)\right]\right)
\end{aligned}
$$

In the above definitions $i=1,2,3,4,5$, the superscript $\mathrm{T}$ means transpose, I means identity matrix and diag means a diagonal matrix. The values of $f_{l}\left(\xi_{0}\right), \ldots, f_{l}\left(\xi_{N}\right), \theta_{l}\left(\xi_{0}\right), \ldots, \theta_{l}\left(\xi_{N}\right), \phi_{l}\left(\xi_{0}\right), \ldots, \phi_{l}\left(\xi_{N}\right)$ can be determine from

$F_{L}=B^{-1} G$

The linear operator is defined as:

$L_{f}\left[f^{\wedge}(\eta ; q), \theta^{\wedge}(\eta ; q), \phi^{\wedge}(\eta ; q)\right]$

$$
\begin{aligned}
&= f^{\prime \prime \prime}+d_{1} f+d_{2} f^{\prime \prime}+d_{3} f^{\prime} \\
&-\left(M+\frac{1}{k_{0}}\right) f^{\prime}+G r \theta+G c \phi \\
& L_{\theta}\left[f^{\wedge}(\eta ; q), \theta^{\wedge}(\eta ; q), \phi^{\wedge}(\eta ; q)\right] \\
& \quad=\left(1+\frac{4}{3} N r\right) \theta^{\prime \prime}+e_{1} f+e_{2} \theta^{\prime}+e_{3} f^{\prime} \\
&+ e_{4} \theta+\operatorname{PrDu} \phi^{\prime}+\operatorname{Pr} \theta \\
&+e_{5} f^{\prime}
\end{aligned}
$$

$L_{\phi}\left[f^{\wedge}(\eta ; q), \theta^{\wedge}(\eta ; q), \phi^{\wedge}(\eta ; q)\right]=\phi^{\prime \prime}+g_{1} f+g_{2} \phi^{\prime}+$

$S c S r \theta^{\prime \prime}-S c f^{\prime} \phi+g_{3} \phi+g_{4} \phi-$

$S c k_{r}^{2}$

The unknown functions are $f^{\wedge}(\eta ; q), \theta^{\wedge}(\eta ; q)$, and $\phi^{\wedge}(\eta ; q)$ and $q \in[0,1]$ an embedding parameter. Also, the nonlinear operators $N_{h \bar{f}}, N_{h \bar{\theta}}$ and $N_{h \bar{\phi}}$ are given by:

$N_{h f}\left[f^{\wedge}(\eta ; q), \theta^{\wedge}(\eta ; q), \phi^{\wedge}(\eta ; q)\right]$

$$
\begin{aligned}
& =f^{\prime \prime \prime}+f f^{\prime \prime}+d_{1} f+d_{2} f^{\prime \prime} f^{\prime} f^{\prime}+d_{3} f^{\prime} \\
& -\left(M+\frac{1}{k_{0}}\right) f^{\prime}+G r \theta+G c \phi
\end{aligned}
$$

$N_{h \theta}\left[f^{\wedge}(\eta ; q), \theta^{\wedge}(\eta ; q), \phi^{\wedge}(\eta ; q)\right]$

$$
\begin{aligned}
& =\left(1+\frac{4}{3} N r\right) \theta^{\prime \prime}+e_{1} f+e_{2} \theta^{\prime}+e_{3} f^{\prime}+e_{4} \theta+\operatorname{PrDu} \phi^{\prime} \\
& +\operatorname{Pr} \theta+e_{5} f^{\prime}+\operatorname{Pr} f \theta^{\prime}-\operatorname{Pr} f^{\prime} \theta \\
& +\operatorname{PrEc} E f^{\prime} f^{\prime} \\
& \begin{aligned}
& N_{h \phi}\left[f^{\wedge}(\eta ; q), \theta^{\wedge}(\eta ; q), \phi^{\wedge}(\eta ; q)\right] \\
& \quad \phi^{\prime \prime}+g_{1} f+g_{2} \phi^{\prime}+S c S r \theta^{\prime \prime}-S c f^{\prime} \phi \\
&+g_{3} \phi+g_{4} \phi-S c k_{r}^{2} \phi+S c f^{\prime} \phi
\end{aligned}
\end{aligned}
$$

Also the zeroth order deformation is given by:

$(1-q) L_{\bar{f}}\left[f^{\wedge}(\eta ; q)-f_{0}^{\wedge}(\eta)\right]=$

$q \hbar_{\bar{f}} H_{\bar{f}}(\eta) N_{h f}\left[f^{\wedge}(\eta ; q), \theta^{\wedge}(\eta ; q), \phi^{\wedge}(\eta ; q)\right]$

$(1-q) L_{\bar{\theta}}\left[\theta^{\wedge}(\eta ; q)-\theta_{0}^{\wedge}(\eta)\right]=$

$q \hbar_{\bar{\theta}} H_{\bar{\theta}}(\eta) N_{h \theta}\left[\begin{array}{llll}f & (\eta ; q), \theta & (\eta ; q), \phi & (\eta ; q)\end{array}\right]$

where $N_{h f}, N_{h \theta}$, and $N_{h \phi}$ is given as defined in (41)-(44) above and $\hbar_{\bar{f}}, \hbar_{\bar{\theta}}$ and $\hbar_{\bar{\phi}}$ are nonzero convergence controlling auxiliary parameters. Differentiating (45)-(47) $m$ times with respect to $q$, setting $q=0$ and divide the result by $m$ ! gives the following $m t h$ order deformation equations.

$$
\begin{aligned}
& L_{\bar{f}}\left[\bar{f}(\xi)-\chi_{m} \bar{f}_{m-1}(\xi)\right] \\
& =\hbar_{\bar{f}} H_{\bar{f}}(\xi) R_{m}^{\bar{f}}(\xi) \\
& L_{\bar{\theta}}\left[\bar{\theta}(\xi)-\chi_{m} \bar{\theta}_{m-1}(\xi)\right]=\hbar_{\bar{\theta}} H_{\bar{\theta}}(\xi) R_{m}^{\bar{\theta}}(\xi) \\
& L_{\bar{\phi}}\left[\bar{\phi}(\xi)-\chi_{m} \bar{\phi}_{m-1}(\xi)\right]=\hbar_{\bar{\phi}} H_{\bar{\phi}}(\xi) R_{m}^{\bar{\phi}}(\xi)
\end{aligned}
$$

subject to

$\bar{f}_{m}(-1)=0 . \bar{\theta}_{m}(-1)=0, \bar{\phi}_{m}(-1) \exists 0$

$\left.\bar{f}_{m}^{\prime}(1)=0, \bar{\theta}_{m}(1)=0, \bar{\phi}_{m}(1)=0\right\}$

given that

$R_{m}^{\bar{f}}(\xi)$

$=f_{m-1}^{\prime \prime \prime}+d_{1} f_{m-1}+d_{2} f_{m-1}^{\prime \prime}+d_{3} f_{m-1}^{\prime}-\left(M+\frac{1}{k_{0}}\right) f_{m-1}^{\prime}$

$+\operatorname{Gr} \theta_{m-1}+\operatorname{Gc} \phi_{m-1}$

$+\sum_{n=0}^{m-1}\left(f_{n}^{\prime} f_{m-1-n}^{\prime}\right.$
$\left.+f_{n} f_{m-1-n}^{\prime \prime}\right)$ 


$$
\begin{aligned}
& R_{m}^{\bar{\theta}}(\xi) \\
& =\left(1+\frac{4}{3} N r\right) \theta_{m-1}^{\prime \prime}+e_{1} f_{m-1}+e_{2} \theta_{m-1}^{\prime}+e_{3} f_{m-1}^{\prime}+e_{4} \theta_{m-1} \\
& +\operatorname{PrDu} \phi_{m-1}^{\prime}+\operatorname{Pr} \gamma \theta_{m-1}+e_{5} f_{m-1}^{\prime} \\
& +\sum_{n=0}^{m-1}\left(\operatorname{Pr} f_{n} \theta_{m-1-n}^{\prime}-\operatorname{Pr} f_{n}^{\prime} \theta_{m-1-n}\right. \\
& \left.+\operatorname{PrEc} f_{n}^{\prime} f_{m-1-n}^{\prime}\right) \\
& R_{m}^{\bar{\phi}}(\xi) \\
& =\phi_{m-1}^{\prime \prime}+g_{1} f_{m-1}+g_{2} \phi_{m-1}^{\prime}+g_{3} \phi_{m-1}+\operatorname{ScSr} \theta_{m-1}^{\prime \prime} \\
& -S c k_{r}^{2} \phi_{m-1} \\
& +\sum_{n=0}^{m-1}\left(S c f_{n} \phi_{m-1-n}^{\prime}-S c f_{n}^{\prime} \phi_{m-1-n}\right. \\
& \left.-S c f_{n}^{\prime} \phi_{m-1-n}\right) \\
& \text { And } \\
& \chi_{m}= \begin{cases}0 & m \leq 0 \\
1 & m>1\end{cases}
\end{aligned}
$$

Chebyshev pseudospectral transformation is applied on (47)(49) and the following results are obtained:

$$
\begin{aligned}
& B \bar{f}_{m}=\left(\chi_{m}+h_{\bar{f}}\right) B \bar{f}_{m-1}-h_{\bar{f}}\left(1-\chi_{m}\right) G+\hbar_{\bar{f}} U_{m-1}^{\bar{f}} \\
& B \bar{\theta}_{m}= \\
& \left(\chi_{m}+h_{\bar{\theta}}\right) B \bar{\theta}_{m-1}-h_{\bar{\theta}}\left(1-\chi_{m}\right) G+\hbar_{\bar{\theta}} U_{m-1}^{\bar{\theta}} \\
& \quad B \bar{\phi}_{m}=\left(\chi_{m}+h_{\bar{\phi}}\right) B \bar{\phi}_{m-1}-h_{\bar{\phi}}\left(1-\chi_{m}\right) G+ \\
& \quad \hbar_{\bar{\phi}} U_{m-1}^{\bar{\phi}}
\end{aligned}
$$

subject to

$$
\begin{aligned}
& U_{m-1}^{\bar{f}}= \sum_{n=0}^{m-1}\left[\left(D f_{n}\right)\left(D f_{m-1-n}\right)+\left(f_{n}\right)\left(D^{2} f_{m-1-n}\right)\right] \\
& U_{m-1}^{\bar{\theta}}= \sum_{n=0}^{m-1}\left[\operatorname{Pr} f_{n}\left(D \theta_{m-1-n}\right)-\operatorname{Pr}\left(D f_{n}\right)\left(\theta_{m-1-n}\right)\right. \\
&\left.+\operatorname{PrEc}\left(D f_{n}\right)\left(D f_{m-1-n}\right)\right]
\end{aligned}
$$

In other for us to implement the boundary condition, first and last rows of $U_{m-1}^{\bar{f}}, U_{m-1}^{\bar{\theta}}, U_{m-1}^{\bar{\phi}}$ and $\mathrm{G}$ as well as the first and last columns of B in (55)-(57) is deleted. Also, the first and last rows of the modified $\mathrm{B}$ matrix on the right hand side of (55)-(57) are set to be zero and the following recursive formulas were obtained

$$
\begin{aligned}
\bar{F}_{m}= & \left(\chi_{m}+\hbar_{\bar{f}}\right) B^{-1} B F_{m-1} \\
& +\hbar_{\bar{f}} B^{-1}\left[U_{m-1}^{\bar{f}}-\left(1-\chi_{m}\right) G\right] \\
\bar{\theta}_{m} & =\left(\chi_{m}+\hbar_{\bar{\theta}}\right) B^{-1} B \theta_{m-1}+\hbar_{\bar{\theta}} B^{-1}\left[U_{m-1}^{\bar{f}}-\left(1-\chi_{m}\right) G\right]
\end{aligned}
$$

$$
\begin{aligned}
\bar{\phi}_{m}=\left(\chi_{m}+\hbar_{\bar{\phi}}\right) B^{-1} B \phi_{m-1} \\
+\hbar_{\bar{\phi}} B^{-1}\left[U_{m-1}^{\bar{f}}-\left(1-\chi_{m}\right) G\right]
\end{aligned}
$$

Higher-order approximations of $\bar{f}_{m}(\xi), \bar{\theta}_{m}(\xi), \bar{\phi}_{m}(\xi)$ for $m \geq 1$ is obtained from the recursive formulas defined in (58)-(60) starting from the initial approximations given by

$f_{0}(\eta)=-f_{w}+1-e^{-\eta}, \theta_{0}(\eta)=\phi_{0}(\eta)=e^{-\eta}$

\section{NUMERICAL RESULTS AND DISCUSSION}

The problem of steady free convective flow embedded in porous medium is considered in this paper. The governing equations for momentum, energy, and concentration are set of partial differential equations which were later transformed into nonlinear coupled ordinary differential equations. The transformed coupled nonlinear coupled ordinary differential equations have been solved by using Spectral Homotopy Analysis Method (SHAM). This method has been used by many authors in literature out of which we mention in this paper those carried out by Motsa et al. (2010); Fagbade et al. (2016); Fagbade et al. (2015). The spectral homotopy analysis method combines the homotopy analysis method with Chebyshev spectral collocation method in its implementation. Our problem is the general form of Rao et al. (2014); Ibrahim and Makinde (2010) when $S r=D u=N r=\gamma=k r=k_{0}=E c=0$.

The effects of viscous flow parameters are displayed in figure 2a-13c. In figure (2a)-(2c) the effect of thermal Grashof number is depicted. It is worth mentioning in this work that in a situation when $G r=0$, it indicates the absence of free convective current. Also, when the thermal Grashof number is high, it indicates that the flow in the boundary layer is laminar and vice versa. It is obvious from figure (2a)(2c) that when the thermal Grashof number is increased, the velocity profile is increased but reduces both the temperature and concentration profiles simultaneously. Figure (3a)-(3c) shows the effect of the mass Grashof number on the velocity, temperature and concentration profiles. As shown in the figure (3a)-(3c), increasing $G c$ increases the velocity in the boundary layer but reduces the temperature and concentration profiles. The effect of the permeability parameter $\left(k_{0}\right)$ is displayed in figure (4a)-(4c).

The result shows that increase in the permeability increases the velocity profile but reduces the temperature profile. Figure (5a)-(5c) shows the effect of Prandtl number on the velocity, temperature and concentration profiles. In figure (5a)-(5c), increasing the Prandtl number reduces the temperature profile. This is because thermal conductivity of the fluid decreases when Prandtl number $(\mathrm{Pr})$ is increased and thereby gives rise to decrease in thermal boundary layer thickness.

The viscous dissipation term is depicted in figure (6a)(6c). The viscous dissipation term connotes the Eckert number $(E c)$. The Eckert number explains the relationship between the energy on motion (kinetic energy) in the flow and the enthalpy. The viscous dissipation term is the 
conversion of the energy on motion (kinetic energy) into internal energy as a result of work done against the viscous fluid stresses. As shown in figure (6a)-(6c), Eckert number increases the temperature profile when increased. Figure (7a)(7c) depict the effect of the Schmidt number on the velocity, temperature and concentration profiles. As expected the Schmidt number altered the concentration profile significantly. Increasing the Schmidt number decreases the concentration profile as shown in figure (7a)-(7c). The effect of the chemical reaction parameter is shown in figure (8a)(8c). As shown in the figure, as the chemical reaction parameter increases, the concentration profile decreases. The effect of the suction velocity $\left(f_{w}\right)$ is shown in figure (9a)(9c).

It is interesting to see that, increasing the suction velocity increases both the temperature and concentration profiles respectively. The effect of the applied transverse magnetic parameter is shown in figure (10a)-(10c). In figure (10a)-(10c), magnetic parameter decreases the velocity profile. Also, increasing magnetic parameter increases both the temperature and concentration profiles respectively. The magnetic field parameter gives rise to a resistive force called
Lorenz force which tends to slow down the motion of an electrically conducting fluid.

Furthermore, the effect of both soret and dufour is depicted in figure (11a)-(11c) and figure (12a)-(12c). We observed from these figures that the effects of soret and dufour are opposite. Soret effect altered the concentration profile while dufour effect alters the temperature profile. Effects of thermal radiation parameter on the velocity, temperature and concentration profiles are displayed in figure (13a)-(13c). Thermal radiation is of great importance when the temperature is high. Thermal radiation enhances convective flow. The thermal condition of the fluid is high when thermal radiation is increased. It is obvious from Table 1 where values of the skin friction coefficient, Nusselt number and Sherwoo number of the present work are compared with (Rao et al, 2014) when $\operatorname{Pr}=0.71, E c=N r=\gamma=k r=k_{0}=0$.

Table 1: Comparison between the values of the skin friction coefficient $\left(F^{\prime \prime}(0)\right)$, Nusselt number $\left(-\theta^{\prime}(0)\right)$ and

Sherwood number $\left(-\phi^{\prime}(0)\right)$ with that of Rao et al. (2014).

\begin{tabular}{|c|c|c|c|c|c|c|c|c|c|c|}
\hline \multirow[b]{2}{*}{$G_{T}$} & \multirow[b]{2}{*}{$G_{C}$} & \multirow[b]{2}{*}{$M$} & \multirow[b]{2}{*}{$f w$} & \multirow[b]{2}{*}{$S c$} & \multicolumn{3}{|c|}{ Rao et al. (2014) } & \multicolumn{3}{|c|}{ Present Work } \\
\hline & & & & & $F^{\prime \prime}(0)$ & $-\theta^{\prime}(0)$ & $-\phi^{\prime}(0)$ & $F^{\prime \prime}(0)$ & $-\theta^{\prime}(0)$ & $-\phi^{\prime}(0)$ \\
\hline 0.1 & 0.1 & 0.1 & 0.1 & 0.62 & 0.889876 & 0.796832 & 0.72667 & 0.88988 & 0.796843 & 0.726676 \\
\hline 05 & 0.1 & 0.1 & 0.1 & 0.62 & 0.696715 & 0.837821 & 0.76632 & 0.696771 & 0.837841 & 0.76846 \\
\hline 1.0 & 0.1 & 0.1 & 0.1 & 0.62 & 0.47561 & 0.875163 & 0.802258 & 0.47626 & 0.875172 & 0.802263 \\
\hline 0.1 & 0.5 & 0.1 & 0.1 & 0.62 & 0.687913 & 0.841779 & 0.770318 & 0.687914 & 0.841782 & 0.770331 \\
\hline 0.1 & 1.0 & 0.1 & 0.1 & 0.62 & 0.458518 & 0.881508 & 0.808662 & 0.458521 & 0.881511 & 0.808671 \\
\hline 0.1 & 0.1 & 1.0 & 0.1 & 0.62 & 1.26709 & 0.721516 & 0.65876 & 1.267199 & 0.72159 & 0.65966 \\
\hline 0.1 & 0.1 & 3.0 & 0.1 & 0.62 & 1.87041 & 0.615411 & 0.562143 & 1.870490 & 0.615423 & 0.562148 \\
\hline 0.1 & 0.1 & 0.1 & 0.1 & 0.62 & 0.571186 & 0.560411 & 0.52824 & 0.571191 & 0.560418 & 0.52844 \\
\hline 0.1 & 0.1 & 0.1 & 3.0 & 0.62 & 0.379154 & 0.396474 & 0.384708 & 0.379163 & 0.396481 & 0.384122 \\
\hline 0.1 & 0.1 & 0.1 & 1.0 & 0.78 & 0.894076 & 0.794386 & 0.834274 & 0.894083 & 0.794394 & 0.834281 \\
\hline 0.1 & 0.1 & 0.1 & 1.0 & 2.62 & 0.912838 & 0.785889 & 1.65018 & 0.912841 & 0.785891 & 1.65023 \\
\hline
\end{tabular}




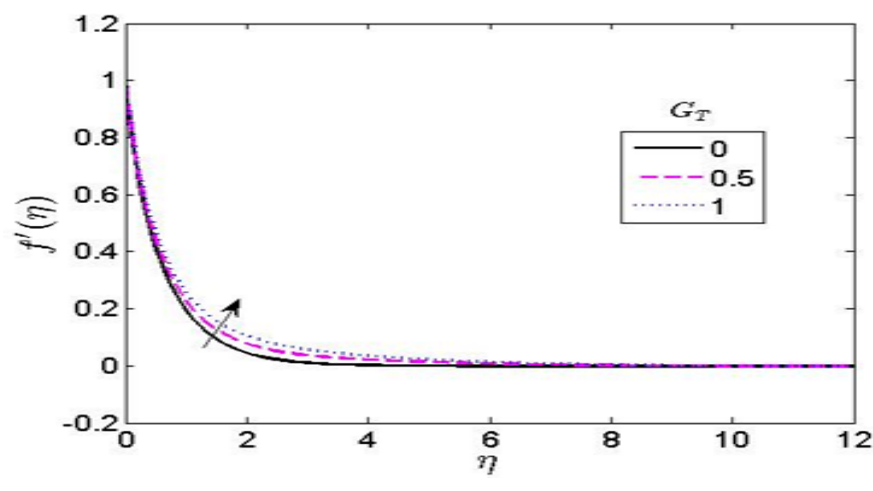

Figure (2a): Velocity profile for different values of $G_{T}$.

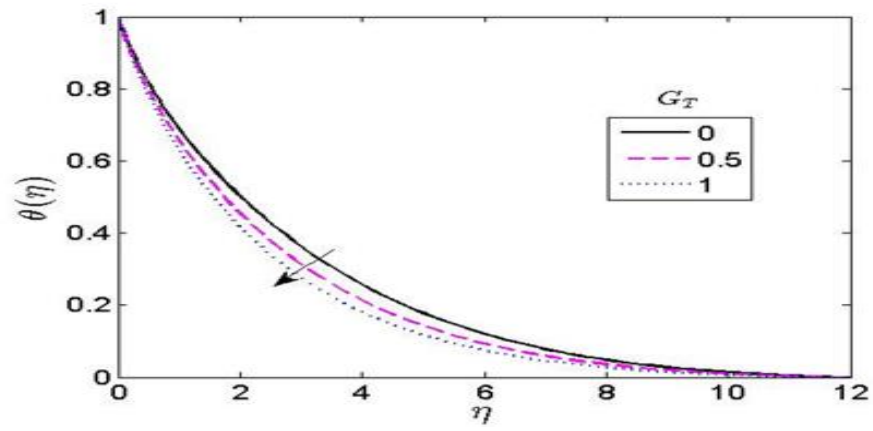

Figure (2b): Temperature profile for different values of $G_{T}$.

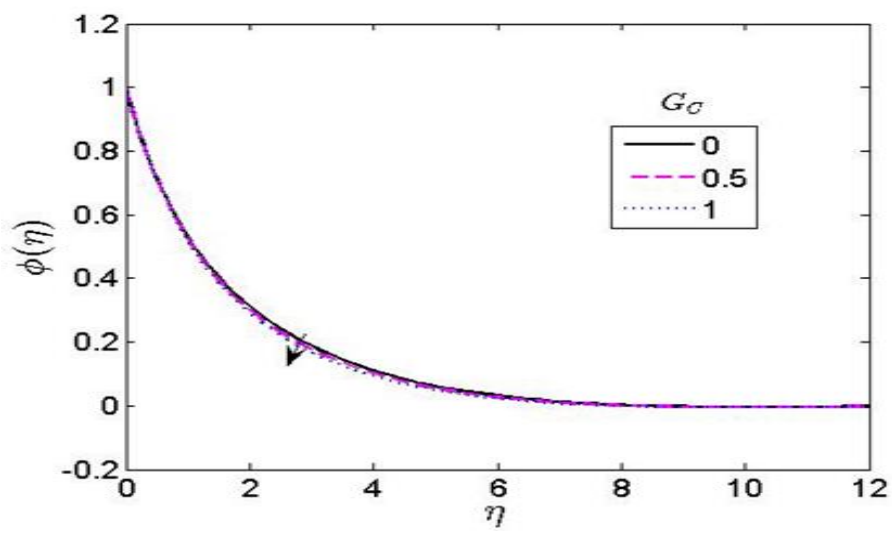

Figure (2c): Concentration profile for different values of $G_{T}$.

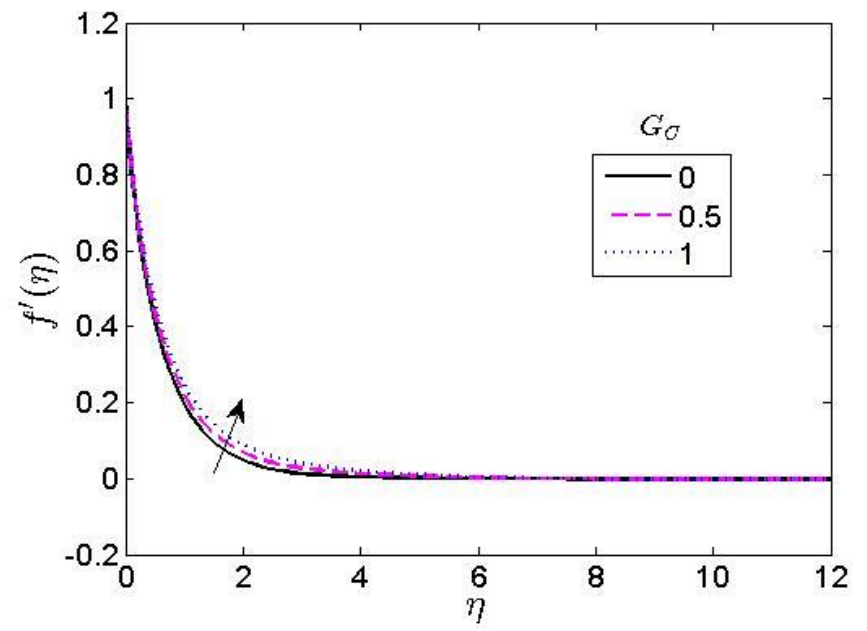

Figure (3a): Velocity profile for different values of $G_{C}$.

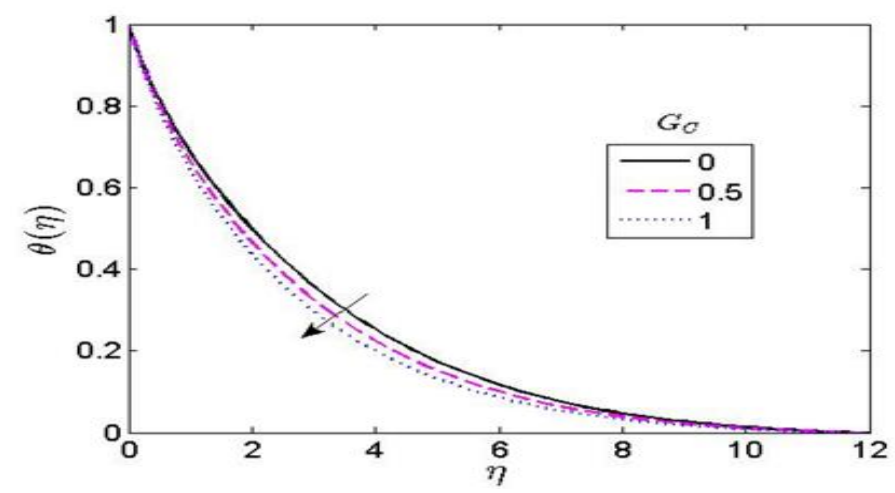

Figure (3b): Temperature profile for different values of $G_{C}$.

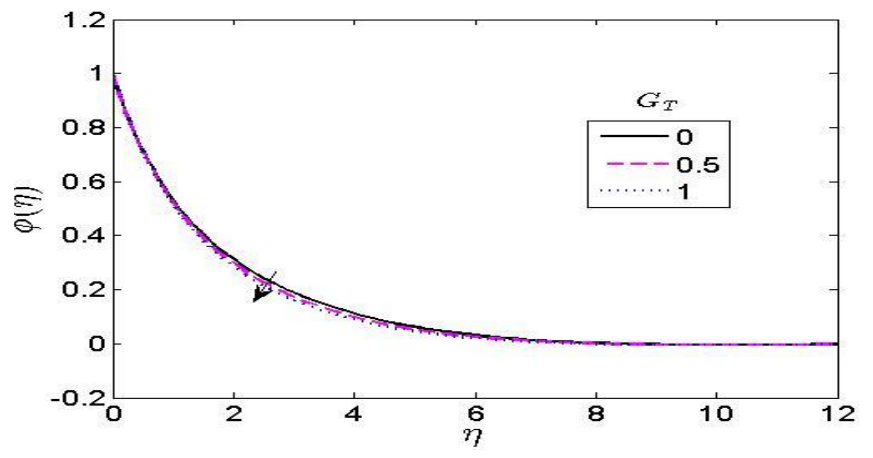

Figure (3c): Concentration profile for different values of $G_{T}$.

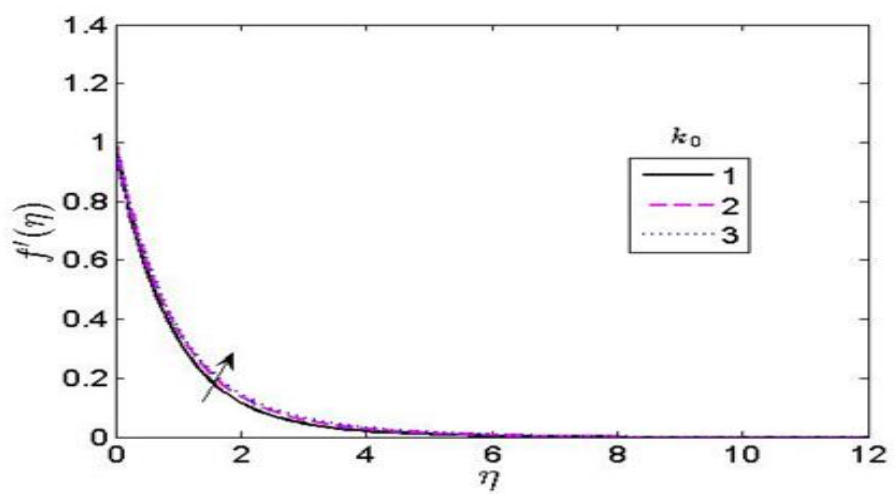

Figure (4a): Velocity profile for difference values of $\boldsymbol{k}_{\boldsymbol{0}}$.

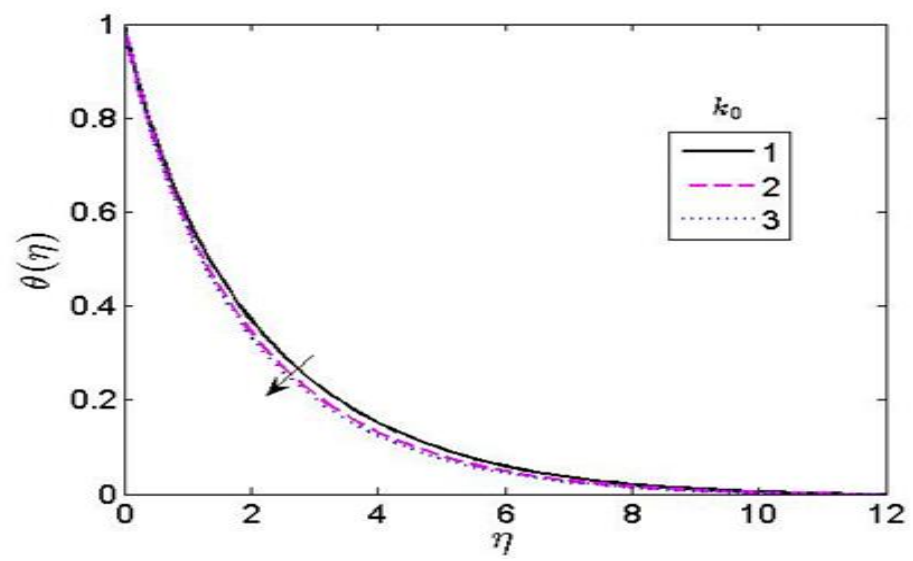

Figure (4b): Temperature profile for different values of $\boldsymbol{k}_{\boldsymbol{l}}$. 


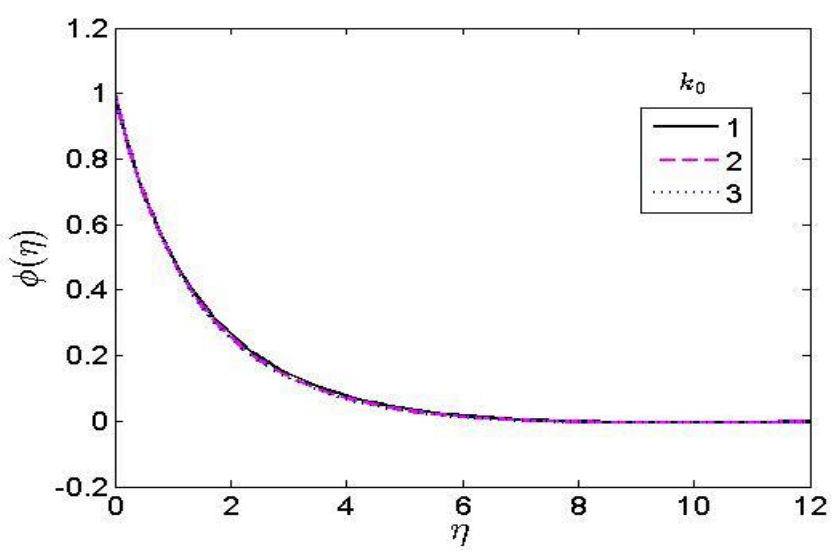

Figure (4c): Concentration profile for different values of $\boldsymbol{k}_{\boldsymbol{0}}$.

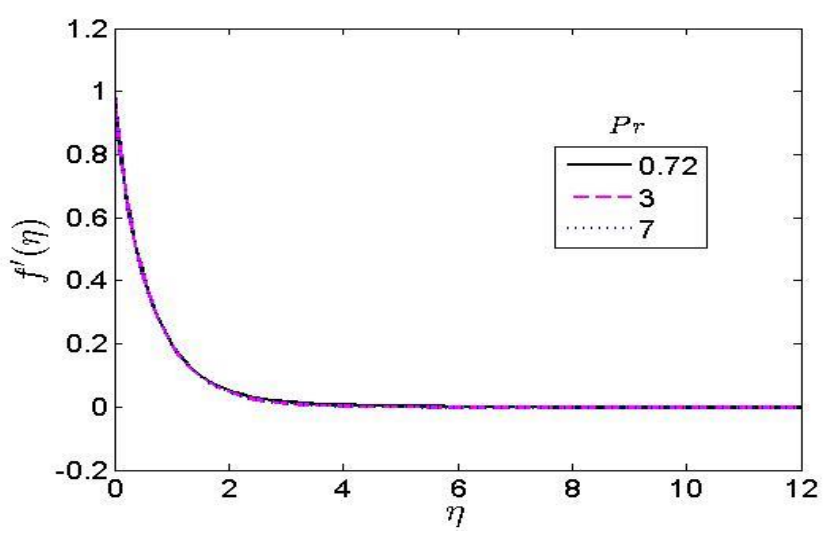

Figure (5a): Velocity profile for different values of $\boldsymbol{P}_{r}$.

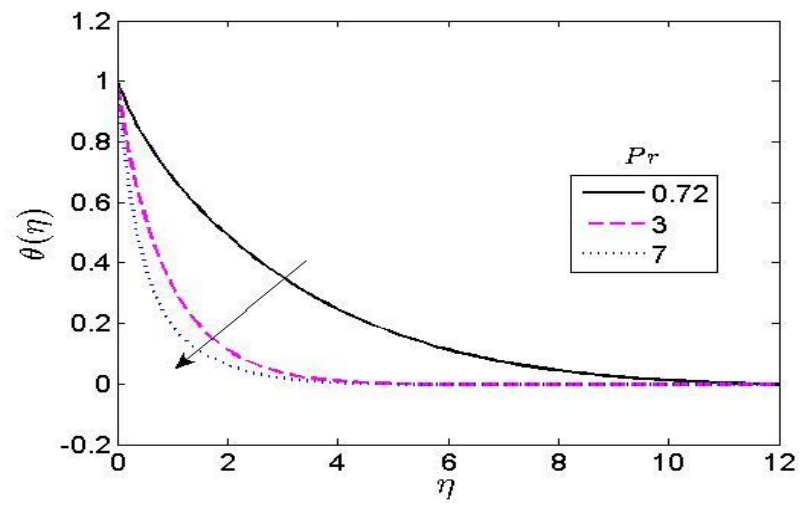

Figure (5b): Temperature profile for different values of $\boldsymbol{P}_{\boldsymbol{r}}$.

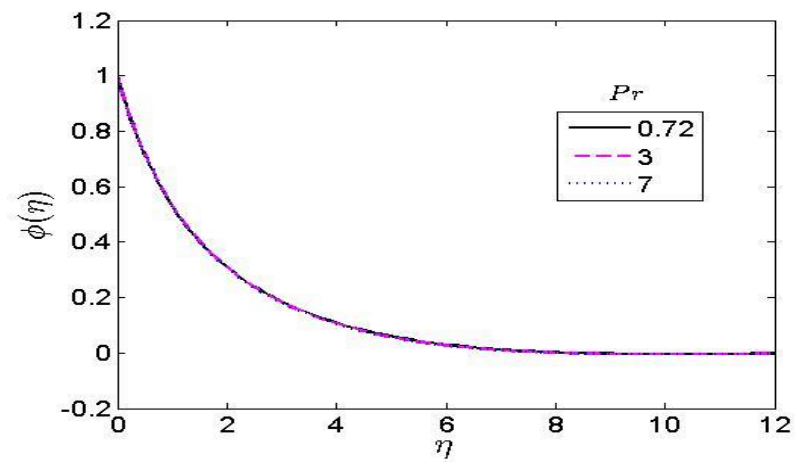

Figure (5c): Concentration profile for different values of $\boldsymbol{P}_{\boldsymbol{r}}$.

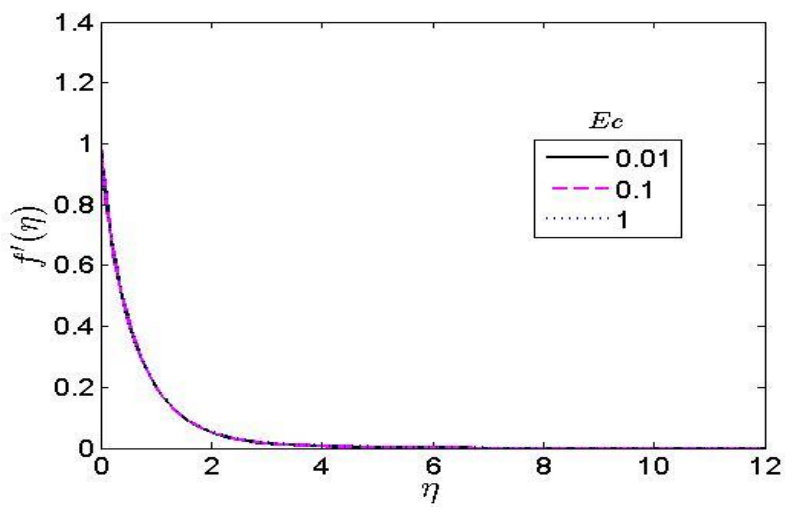

Figure (6a): Velocity profile for different values of $E_{C}$.

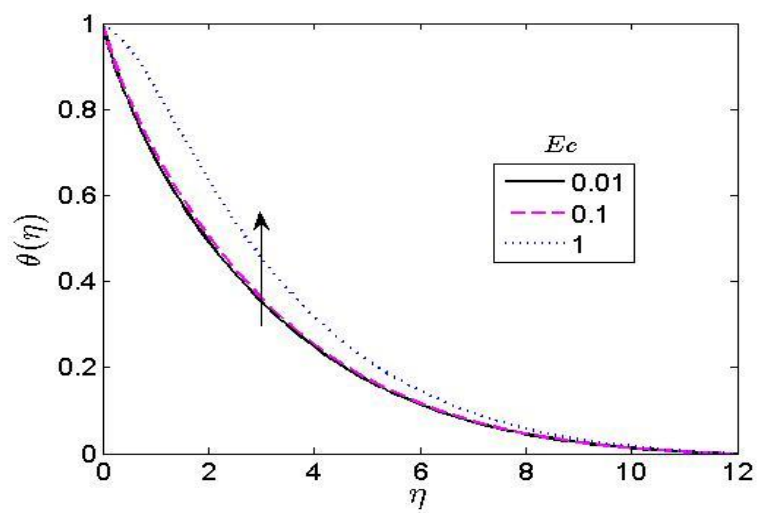

Figure (6b): Temperature profile for different values of $E_{C}$.

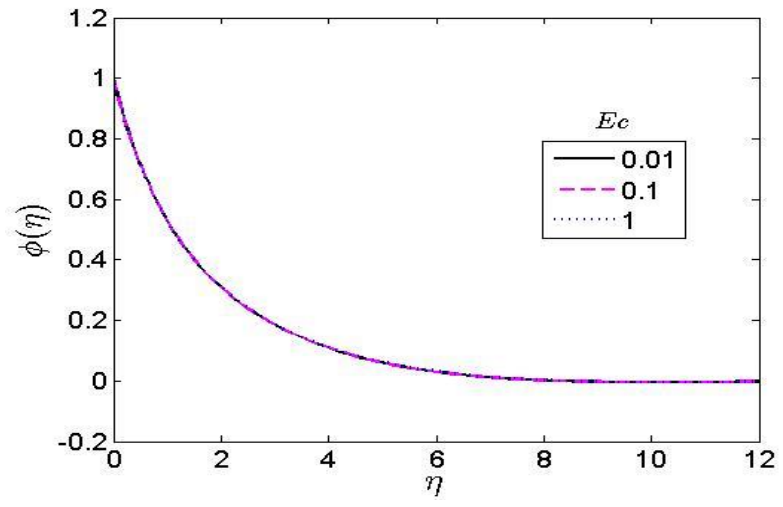

Figure (6c): Concentration profile for different values of $E$ r.

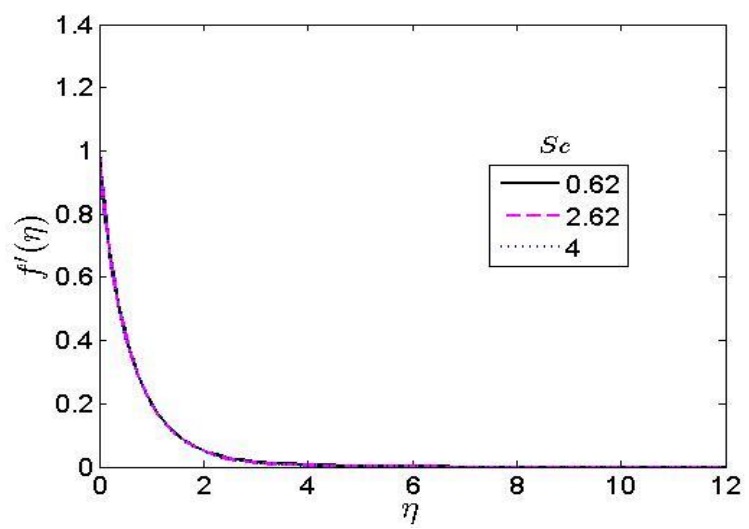

Figure (7a): Velocity profile for different values of $S_{C}$. 


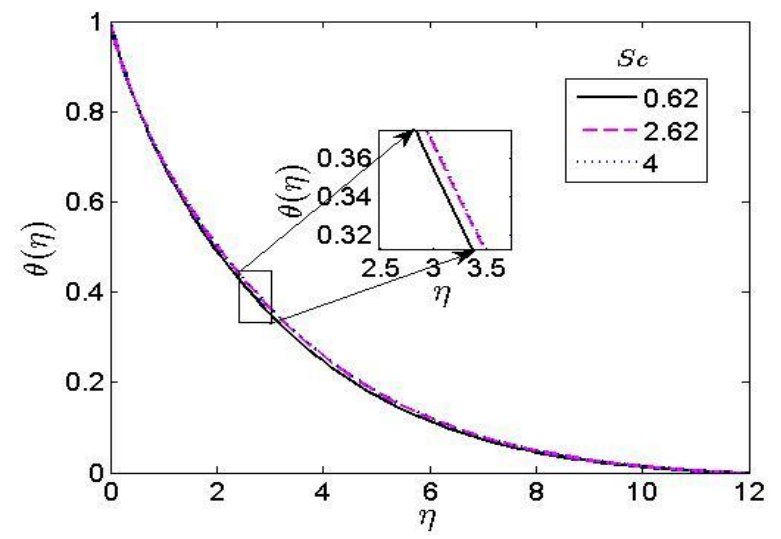

Figure (7b): Temperature profile for different values of $S_{C}$.

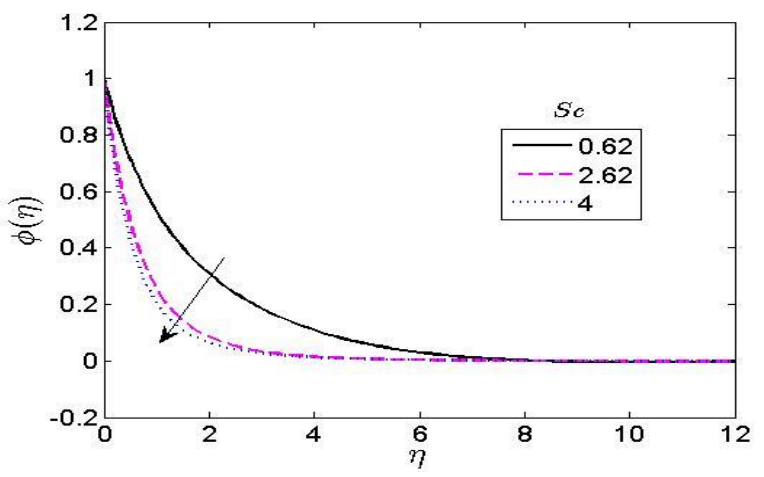

Figure (7c): Concentration profile for different values of $S_{C}$.

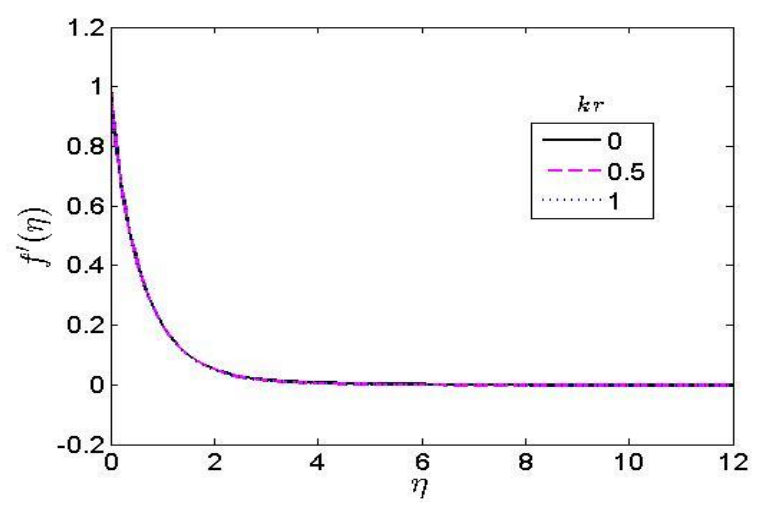

Figure (8a): Velocity profile for different values of $\boldsymbol{k}_{\boldsymbol{r}}$.

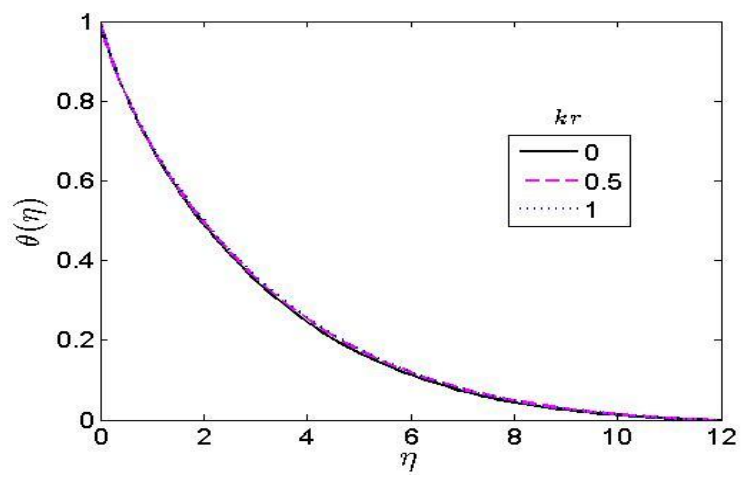

Figure (8b): Temperature profile for different values of $k_{r}$.

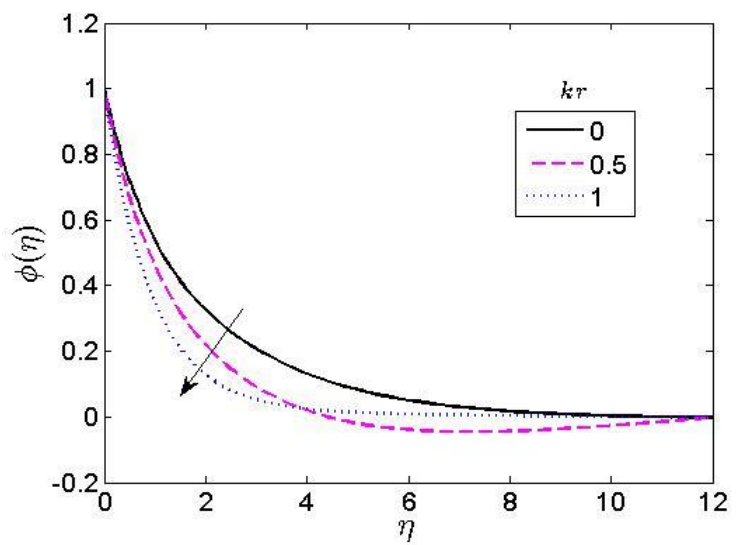

Figure (8c): Concentration profile for different values of $\boldsymbol{k}_{\boldsymbol{r}}$.

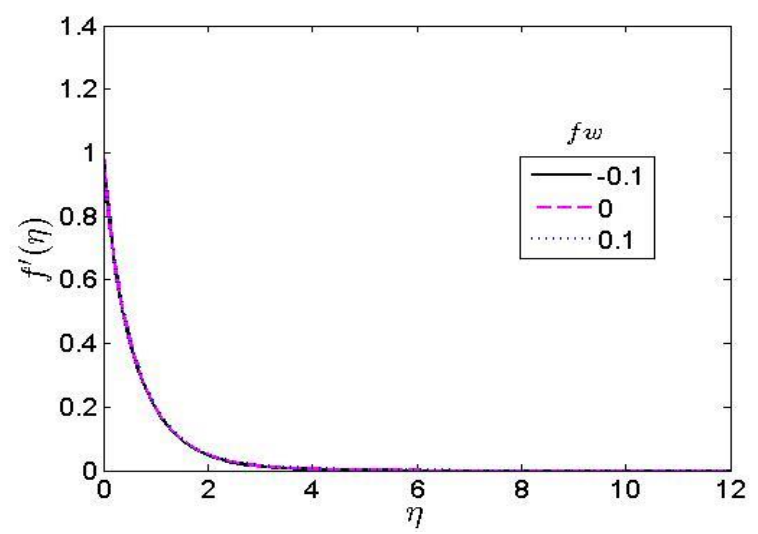

Figure (9a): Velocity profile for different values of $f_{w}$.

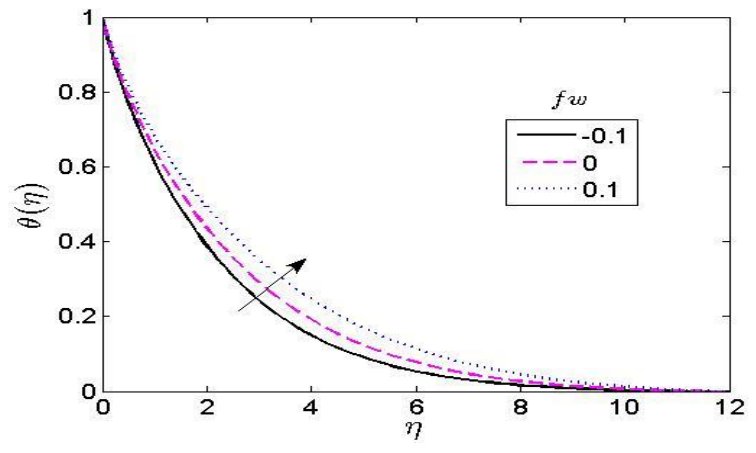

Figure (9b): Temperature profile for different values of $f_{w}$.

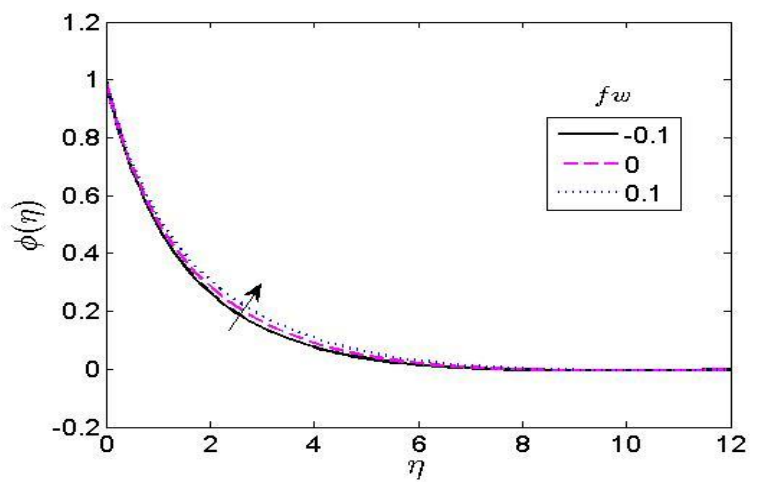

Figure (9c): Concentration profile for different values of $f_{w}$. 


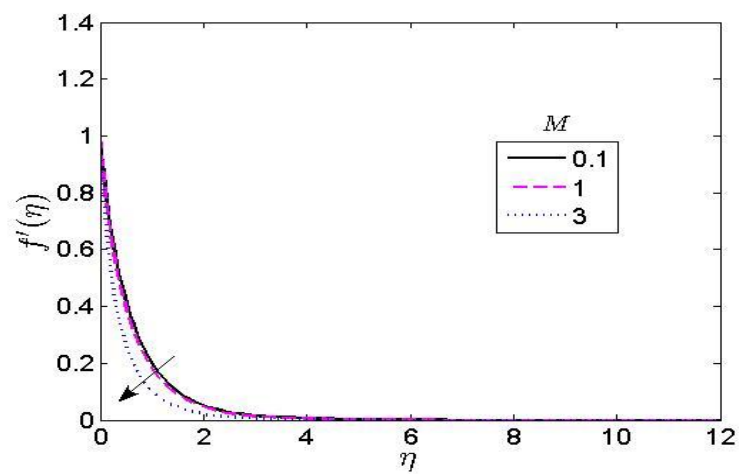

Figure (10a): Velocity profile for different values of $M$.

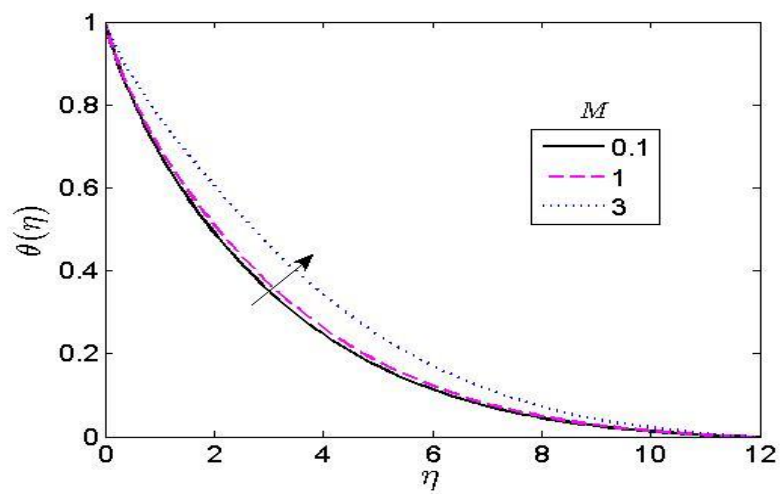

Figure (10b): Temperature profile for different values of $M$.

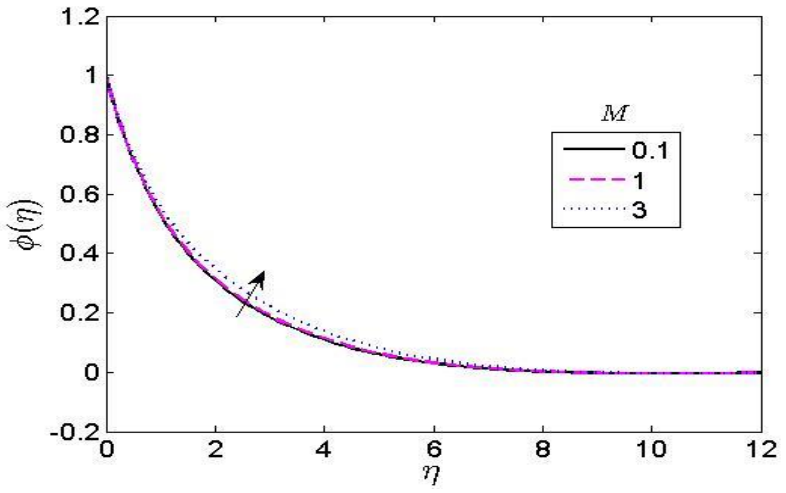

Figure (10c): Concentration profile for different values of $M$.

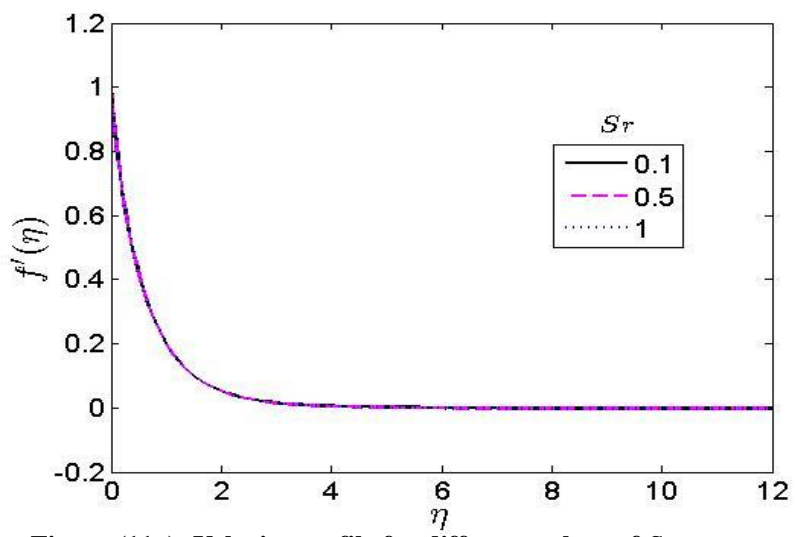

Figure (11a): Velocity profile for different values of $S_{r}$.

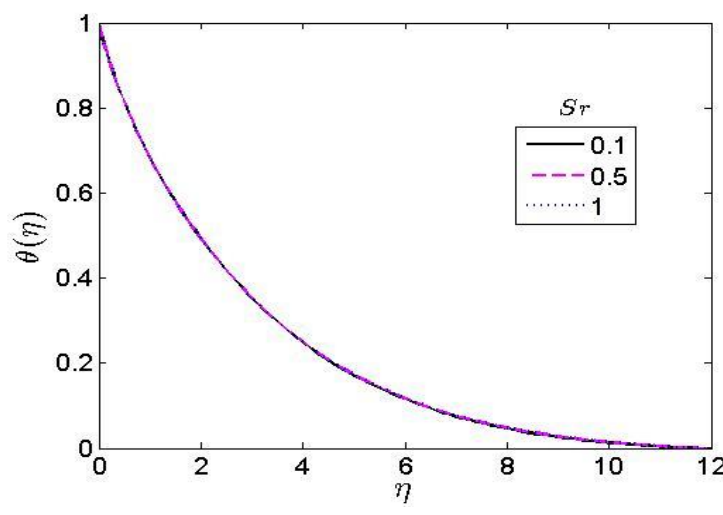

Figure (11b): Temperature profile for different values of $S_{r}$.

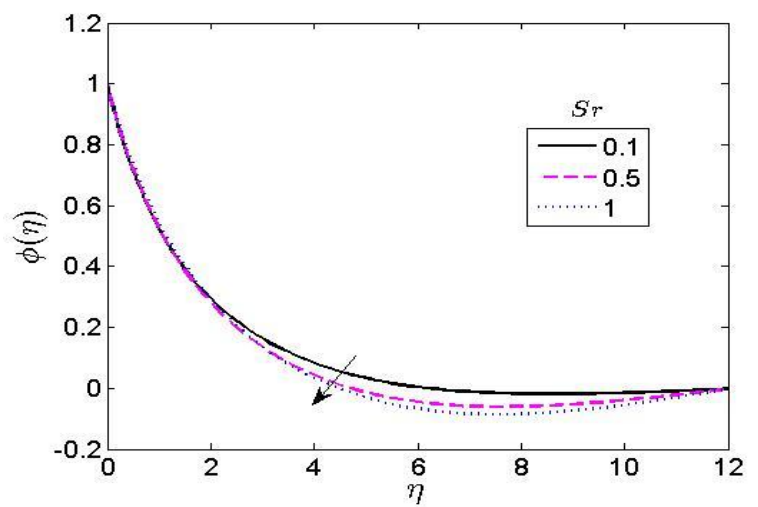

Figure (11c): Concentration profile for different values of $S_{r}$.

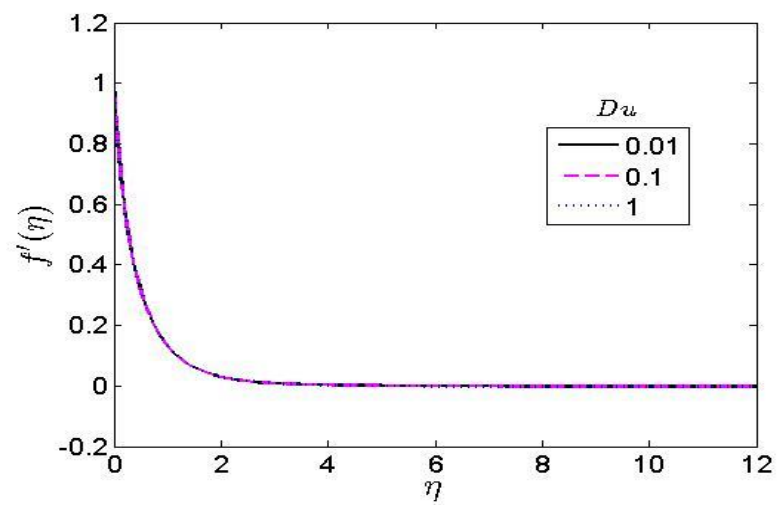

Figure (12a): Velocity profile for different values of $D_{u}$.

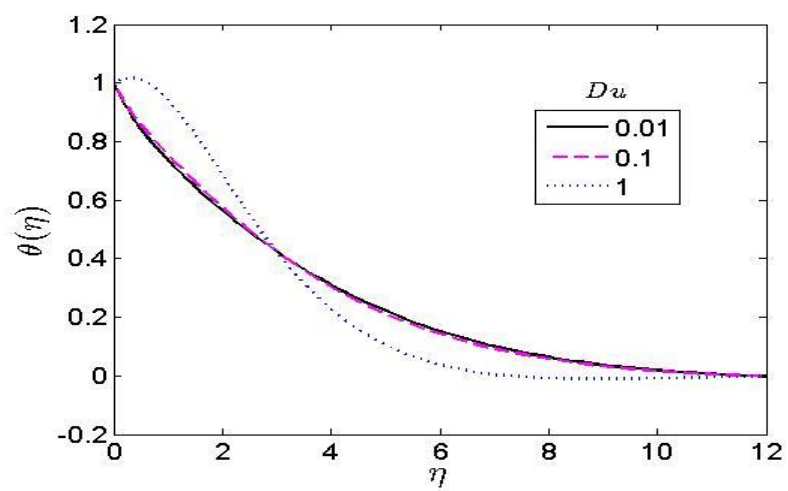

Figure (12b): Temperature profile for different values of $D_{u}$. 


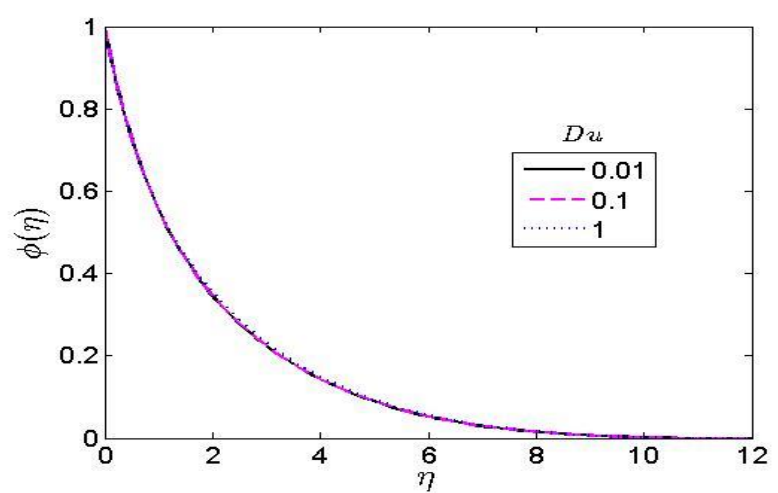

Figure (12c): Concentration profile for different values $D_{u}$.

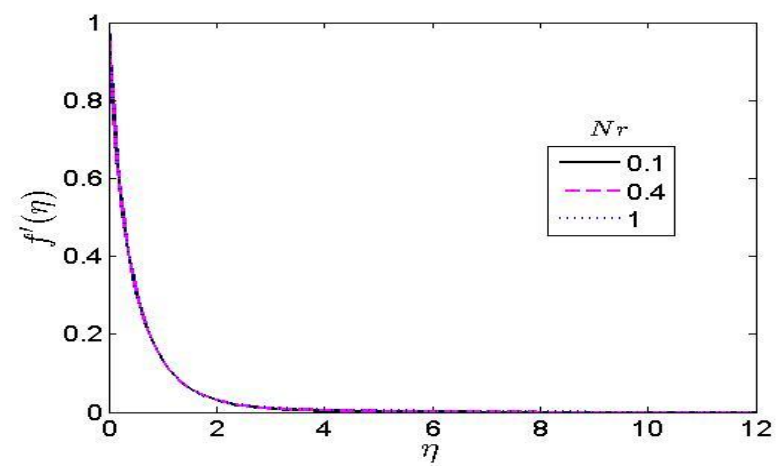

Figure (13a): Velocity profile for different values of $N_{r}$.

\section{$\mathrm{V}$ CONCLUSION}

This paper studied influence of magnetic field and thermal radiation on steady free convective flow embedded in a porous medium with Soret and Dufour effects. The problem under investigation was solved using the spectral homotopy analysis method (SHAM). Numerical computations were done and the following conclusions were drawn from the results obtained. Increasing the Prandtl number decreases the temperature profile. The suction velocity intensifies the temperature and concentration profiles but negligible on the velocity profile when it is increased.

The viscous dissipation term namely Eckert number. Increasing the magnetic parameter reduces the velocity profile due to the applied magnetic field in the opposite direction to the flow gives rise to a resistive force which tends to slow down the motion of an electrically conducting fluid. Interestingly increasing the magnetic parameter increases both the temperature and concentration profiles respectively. It is noticed that increasing the Soret number decreases far away from the plate but negligible or has no effect very close to the plate. The thermal radiation parameter intensifies the temperature profile when increased.

\section{REFERENCES}

Alao F.I.; A.I. Fagbade; B.O. Falodun. (2016). Effects of thermal radiation, soret and dufour on an unsteady heat and mass transfer flow of a chemically reacting fluid past a semiinfinite vertical plate with viscous dissipation. Journal of the Nigerian Mathematical Society, 35: 142-158.

Bhavana M.; D. Chenna Kesavaiah; A. Sudhakaraiah (2013). The soret effect on free convective unsteady MHD

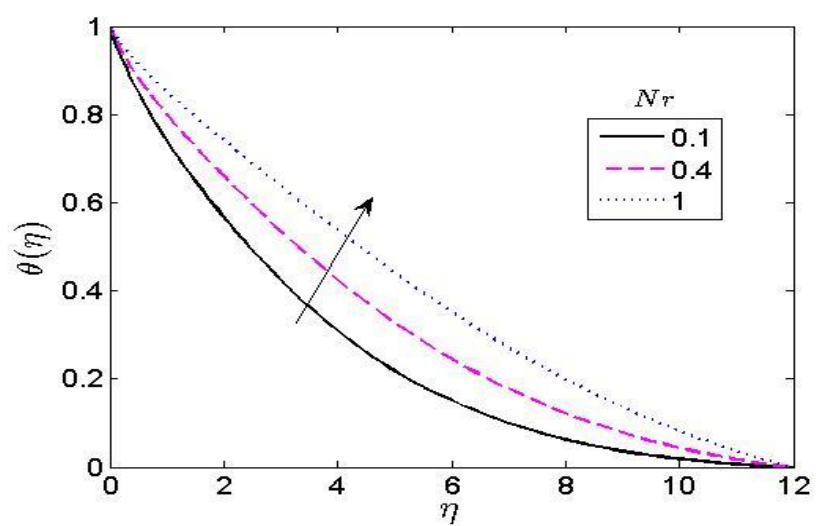

Figure (13b): Temperature profile for different values of $N_{r}$.

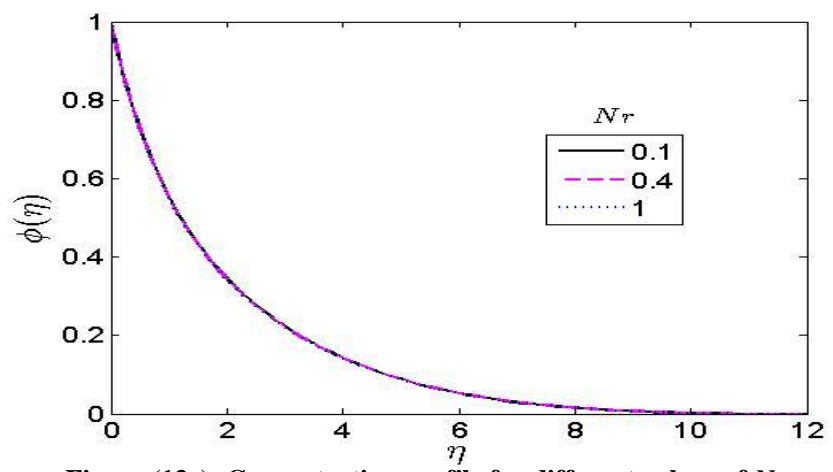

Figure (13c): Concentration profile for different values of $N_{r}$.

flow over a vertical plate with heat source. International Journal of Innovative Research in Science, Engineering and Technology, 2(5): 1617-1628.

Fagbade A.I.; B.O. Falodun; C.U. Boneze. (2015). Influence of magnetic field, viscous dissipation and thermophoresis on Darcy-Forcheimer mixed convection flow in fluid saturated porous media. American Journal of Computational Mathematics, 5: 18-40.

Fagbade A.I.; B.O. Falodun; A.J. Omowaye. (2016). MHD natural convection flow of viscoelastic fluid over an accelerating permeable surface with thermal radiation and heat source or sink:spectral homotopy analysis approach. Ain Shams Engineering Journal (Article in press).

Ibrahim S.M. (2014). Unsteady MHD free convective flow along a vertical porous plate embedded in a porous medium with heat generation, variable suction and chemical reaction effects. Chemical and Process Engineering Research, 21: 86-97.

Ibrahim S.Y. and Makinde O.D. (2010). Chemically reacting MHD boundary layer flow of heat and mass transfer over a moving vertical plate with suction. Scientific Research and Essays. 5(19): 2875-2882.

Ibrahim S.Y. and Makinde O.D. (2011). Chemically reacting Magnetohydrodynamics (MHD) boundary layer flow of heat and mass transfer past a low-heat resistant sheet moving vertically downwards. Scientific Research and Essays, 6(22): 4762-4775.

Kala, B. S.; M.S. Rawat; G. V. R. Reddy and N. Rawat. (2017). Diffusion-thermo and thermo-diffusion effects on MHD fluid flow over Non-linearly stretching sheet 
through a Non-Darcy porous medium. Asian Research Journal of Mathematics, 2(2): 1-17.

Kalyani C.; M. Chenna; Krishna Reddy and N. Kishan (2015). MHD mixed convection flow past a vertical porous plate in a porous medium with heat source/sink and soret effects. American Chemical Science Journal, 7(3): 150159.

Liao, S.J. (2003). Beyond Perturbation: Introduction to the homotopy analysis method. Chapman and Hall/CRC Press Boca.

Liao S.J. (2012). Homotopy Analysis method in nonlinear differential equations. Springer and higher education press, Berlin and Beijing. http://dx.doi.org/10.1007/978-3-642-25132-0.

Mahdy A. (2008). The effects of radiation on unsteady MHD convective heat transfer past a semi-infinite vertical porous moving surface with variable suction. Latin American Applied Research, 38: 337-344.

Mohamed R.A.; S.M. Abo-Dahab and T.A. Notal (2010). Thermal Radiation and MHD effects on free convective flow of a polar fluid through a porous medium in the presence of internal heat generation and chemical reaction. Mathematical Problems in Engineering, 1-27.

Motsa S.S.; P. Sibanda; S. Stateyi (2010). A new spectral homotopy analysis method for solving a nonlinear second order BVP. Commun Nonlinear Sci Numer Simulate, 15: 2293-2302.

Olanrewaju P.O. (2012). Similarity solution for natural convection from a moving vertical plate with internal heat generation and a convective boundary condition in the presence of thermal radiation and viscous dissipation. Report and Opinion, 4(8): 68-76.

Prakash, J.; P. Durga Prasad; R.V.M.S.S. Kiran Kumar and S.V.K. Varma (2016). Diffusion-thermo effects on MHD free convective radiative and chemically reactive boundary layer flow through a porous medium over a vertical plate. Journal of computational and Applied Research in Mechanical Engineering, 5(2): 11-126.

Rajput U.S., and S. Kumar (2012). Radiation effects on MHD flow past an impulsively started vertical plate with variable heat and mass transfer. International Journal of Applied Mathematics and Mechanics 8(1), 66-85.

Rao Srinivasa G.; B. Ramana; B. Rami Reddy; G. Vidyasagar (2014). Soret and Dufour effects on MHD boundary layer flow over a moving vertical porous plate with suction. International Journal of Emerging Trends in Engineering and Development, 2(4): 215-226.

Reddy Ramana G.V.; S. Mohammed Ibrahim; and V.S. Bhagavan (2014). Similarity transformation of heat and mass transfer effects on steady MHD free convective dissipative fluid flow past an inclined porous surface with chemical reaction. Journal of Naval Architecture and Marine Engineering, 11: 157-166

Rushikumar B. and Gangadhar K. (2012). MHD free convection flow between two parallel porous walls with varying temperature. International Journal of Engineering, 67-72.

Sharma Ram Bishwa and Aich Animesh (2016). Soret and Dufour effects on steady MHD flow in presence of heat source through a porous medium over a non-isothermal stretching sheet. IOSR Journal of Mathematics.

Sravanthi C.S.; A. Leela ratnam and N. B. Reddy. (2013). Thermo-diffusion and chemical reaction effects on a steady mixed convective heat and mass transfer flow with induced magnetic field. International Journal of Innovative Research in Science, Engineering and Technology, 2(9): 4415-4424.

Subhakar M.K., and Gangadhar K. (2012). Soret and Dufour effects on MHD free convection heat and mass transfer flow over a sretching vertical plate with suction and heat source/sink. International Journal of Modern Engineering Research, 2(5): 3458-3468. 